\title{
Towards Personalized Auditory Models: Predicting Individual Sensorineural Hearing-Loss Profiles From Recorded Human Auditory Physiology
}

Trends in Hearing

Volume 25: 1-22

(C) The Author(s) 2021

Article reuse guidelines:

sagepub.com/journals-permissions DOI: $10.1177 / 2331216520988406$ journals.sagepub.com/home/tia

@SAGE

\author{
Sarineh Keshishzadeh' (D, Markus Garrett ${ }^{2}$ (D), and Sarah Verhulst'
}

\begin{abstract}
Over the past decades, different types of auditory models have been developed to study the functioning of normal and impaired auditory processing. Several models can simulate frequency-dependent sensorineural hearing loss (SNHL) and can in this way be used to develop personalized audio-signal processing for hearing aids. However, to determine individualized SNHL profiles, we rely on indirect and noninvasive markers of cochlear and auditory-nerve (AN) damage. Our progressive knowledge of the functional aspects of different SNHL subtypes stresses the importance of incorporating them into the simulated SNHL profile, but has at the same time complicated the task of accomplishing this on the basis of noninvasive markers. In particular, different auditory-evoked potential (AEP) types can show a different sensitivity to outer-hair-cell $(\mathrm{OHC})$, inner-hair-cell $(\mathrm{IHC})$, or AN damage, but it is not clear which AEP-derived metric is best suited to develop personalized auditory models. This study investigates how simulated and recorded AEPs can be used to derive individual AN- or $\mathrm{OHC}$-damage patterns and personalize auditory processing models. First, we individualized the cochlear model parameters using common methods of frequency-specific OHC-damage quantification, after which we simulated AEPs for different degrees of AN damage. Using a classification technique, we determined the recorded AEP metric that best predicted the simulated individualized cochlear synaptopathy profiles. We cross-validated our method using the data set at hand, but also applied the trained classifier to recorded AEPs from a new cohort to illustrate the generalizability of the method.
\end{abstract}

\section{Keywords}

individualized hearing-loss profile, envelope following response, cochlear synaptopathy, sensorineural hearing loss, auditory modeling, electrophysiology, auditory-evoked potentials

Received 7 July 2020; Revised 13 November 2020; accepted 21 December 2020

Auditory-evoked potentials (AEPs) are widely adopted as markers of sensorineural hearing loss (SNHL) in clinical and research settings. In research animals, auditory brainstem response (ABR) or envelope-following response (EFR) amplitudes can be used to quantify auditory-nerve (AN) fiber damage, that is, cochlear synaptopathy (CS; Furman et al., 2013; Kujawa \& Liberman, 2009; Sergeyenko et al., 2013; Shaheen et al., 2015). However, applying the same AEP markers for CS diagnosis in humans has yielded mixed success, since AEP amplitudes can be affected by (a) other coexisting SNHL aspects such as outer-hair-cell (OHC) damage (Chen et al., 2008; Don \& Eggermont, 1978; Garrett \& Verhulst, 2019; Gorga et al., 1985; Herdman \& Stapells, 2003; Keshishzadeh et al., 2020;
Verhulst et al., 2016) and (b) subject-specific factors such as age, gender, and head size (Hickox et al., 2017; Mitchell et al., 1989; Trune et al., 1988). Moreover, the sensitivity of AEPs to different degrees of OHC loss and $\mathrm{CS}$ is unclear, and a direct quantification of AN fiber

\footnotetext{
'Hearing Technology @ WAVES, Department of Information Technology, Ghent University, Belgium

${ }^{2}$ Medizinische Physik and Cluster of Excellence Hearing4all, Department of Medical Physics and Acoustics, University of Oldenburg, Oldenburg, Germany

Corresponding Author:

Sarineh Keshishzadeh, Hearing Technology @ WAVES, Department of Information Technology, Ghent University, Technologiepark 126,

Zwijnaarde 9052, Belgium.

Email: sarineh.keshishzadeh@ugent.be
} 
damage through histopathology is impossible in live humans (Bharadwaj et al., 2014). These problems hinder the study of the specific impact of $\mathrm{OHC}$ damage and $\mathrm{CS}$ on recorded AEPs and render an AEP-based quantification of AN fiber damage difficult in listeners with mixed hearing pathologies. However, this last step is crucial when developing personalized models of auditory processing for use within numerical closed-loop hearing restoration systems.

Even though several auditory models incorporate sources of SNHL (e.g., Ewert \& Dau, 2000; Ewert et al., 2013; Heinz et al., 2001; Jepsen \& Dau, 2011; Jepsen et al., 2008; Rohdenburg et al., 2005; Verhulst et al., 2018; Zilany \& Bruce, 2006), methods to individualize the AN-damage pattern on the basis of recorded AEP metrics are nonexistent. Here, we investigate the potential of common AEP markers to individualize the frequency-specific AN-damage profile of personalized auditory models with or without other co-occurring aspects of SNHL. Specifically, we present a combined experimental-modeling method in which (a) individual cochlear-gain-loss (CGL) parameters are extracted from either the audiogram or distortion-product otoacoustic emissions (DPOAEs) and (b) a feature set of recorded AEP metrics is compared to simulated AEP metrics to derive periphery models with different CS profiles. Using a classifier that was trained on simulated AEPs for different SNHL profiles, we selected the individual $\mathrm{AN}$ profile that best explained the recorded AEP features from a test subject. We tested this method on 35 participants, which were separated into groups of young normal-hearing $(\mathrm{yNH})$, older normal-hearing $(\mathrm{oNH})$, and older hearing-impaired $(\mathrm{oHI})$ listeners (Garrett et al., 2020). Validation of our method to predict individual AN-damage profile from recorded AEPs was performed on data from a new cohort.

Before we describe the classification method in detail, we summarize which AEP markers are promising to include. Among the hitherto proposed AEP-derived metrics of AN damage, the ABR wave-I is known to degrade as a consequence of CS in subjects with intact sensory hair cells (Kujawa \& Liberman, 2009; Parthasarathy \& Kujawa, 2018); however, this metric is highly variable in humans (Plack et al., 2016; Stamper \& Johnson, 2015) when the contribution of between-subject variability sources such as head size or tissue resistance are not considered (Prendergast et al., 2018). Even though we can assume that any hearing deficit reflecting on the ABR wave-I would travel through the auditory pathway to reflect on the ABR wave- $\mathrm{V}$ as well, homeostatic gain changes between AN fibers and inferior colliculus (IC) may affect the wave- $\mathrm{V}$ amplitude (Chambers et al., 2016; Henry \& Abrams, 2018; Möhrle et al., 2016; Schaette \& McAlpine, 2011) and hence its diagnostic power for CS diagnosis. Another AEP marker, the EFR amplitude, which reflects the strength of a phase-locked AEP response to an amplitudemodulated (AM) stimulus, was shown to degrade as a consequence of $\mathrm{CS}$ in mice histological studies (Parthasarathy \& Kujawa, 2018; Shaheen et al., 2015) and as a consequence of age in human listeners (Goossens et al., 2016; Vasilkov et al., 2021). EFRs offer a more robust measure of the AN fiber population than the ABR wave-I, when recorded in the same animals (Parthasarathy \& Kujawa, 2018; Plack et al., 2016; Shaheen et al., 2015). However, similar to the ABR wave-V, EFR generators have latencies associated with IC processing (Purcell et al., 2004), thus differences in central auditory processing may reflect on the EFR magnitude to mask individual synaptopathy differences (Chambers et al., 2016; Möhrle et al., 2016; Parthasarathy et al., 2019a, 2019b). To address these issues, relative EFR and ABR metrics were proposed in several studies to cancel out subject-specific factors and isolate the CS component of SNHL in listeners with coexisting OHC-loss: ABR wave-I amplitude growth as a function of stimulus intensity (Furman et al., 2013), ABR wave-I-V latency difference (Coats \& Martin, 1977; Elberling \& Parbo, 1987; Watson, 1996), the wave-V and I amplitude ratio ( $\mathrm{Gu}$ et al., 2012; Hickox \& Liberman, 2014; Schaette \& McAlpine, 2011), EFR amplitude slope as a function of modulation depth (Bharadwaj \& ShinnCunningham, 2014; Guest et al., 2018), the derivedband EFR (Keshishzadeh et al., 2020), or the combined use of the ABR wave-V and EFR (Vasilkov \& Verhulst, 2019). Although these relative metrics are promising, it is not known how OHC loss and CS differentially impact AEPs. Recent modeling approaches have shown promise to design EFR stimuli which are maximally sensitive to $\mathrm{CS}$ in the presence of OHC damage (Vasilkov et al., 2021), but conclusive histopathological evidence is to date not available. To make use of the listed metrics to build personalized hearing profiles for a broad population with various SNHL etiologies, two requirements need to be fulfilled. We need to (a) use AEP markers that are maximally sensitive to the CS aspect of SNHL and (b) combine them with a sensitive marker of $\mathrm{OHC}$ deficits to individualize the $\mathrm{OHC}$ and $\mathrm{CS}$ aspects of SNHL. We thus considered various AEP markers (a total of 13) encompassing spectral magnitudes, timedomain peaks, latencies and relative metrics, and combinations thereof, to identify which markers best predict the simulated individualized CS profiles and can be used for reliable auditory profiling.

\section{Experimental Design}

$\mathrm{ABR}, \mathrm{EFR}$, and OHC-damage markers were derived from recordings of two experimental setups in different 
locations. These recordings were used for development and validation of the proposed method, respectively.

\section{Participants}

The dataset that was used to develop the auditory profiling method included recordings from a total of 43 subjects. They were recruited into three groups: 15 young normal-hearing (yNH: $24.53 \pm 2.26$ years, 8 female), 16 older normal-hearing (oNH: $64.25 \pm 1.88$ years, 8 female) and 12 older hearing-impaired (oHI: $65.33 \pm$ 1.87 years, 7 female) groups. Two oNH subjects were omitted from our study due to nonidentifiable ABR waveforms. The hearing thresholds of the participants were assessed at 12 standard audiometric frequencies between 0.125 and $10 \mathrm{kHz}$ (Auritec AT900, Hamburg, Germany audiometer). AEP stimuli were presented monaurally to the ear with the best $4 \mathrm{kHz}$ threshold. Audiometric thresholds were below $20 \mathrm{~dB}-\mathrm{HL}$ at all measured frequencies in the yNH group and below $25 \mathrm{~dB}-\mathrm{HL}$ for frequencies up to $4 \mathrm{kHz}$ in the oNH group. The oHI listeners had sloping high-frequency audiograms with $4-\mathrm{kHz}$ thresholds above $25 \mathrm{~dB}-\mathrm{HL}$ (Figure 1A). The AEP recordings were conducted in an electrically and acoustically shielded booth, while subjects were sitting in a comfortable chair and watching silent movies.

The second experiment, which was used to validate our method on a new cohort, had 19 yNH subjects, aged between 18 and 25 years ( $21.6 \pm 2.27$ years, 12 female).
Volunteers with a history of hearing pathology or ear surgery were excluded based on a recruitment questionnaire. Audiograms were measured in a double-wall sound-attenuating booth, using an Interacoustics Equinox Interacoustics audiometer. All participants had audiometric thresholds below $25 \mathrm{~dB}-\mathrm{HL}$ within the measured frequency range, that is, 0.125 to $10 \mathrm{kHz}$, and the best ear was determined on the basis of their audiogram and tympanogram. The experiment protocol included AEP measurements with a maximum duration of 3 hours, and we only considered one AEP metric for validation purposes in the present study. AEP recordings were conducted in a quiet room while subjects were seated in a comfortable chair and watching muted movies. To minimize the noise intrusion level, both ears were covered with earmuffs and all electrical devices other than the measurement equipment (Intelligent Hearing Systems) were turned off and unplugged.

Participants of both experiments were informed about the experimental procedure according to the ethical guidelines at Oldenburg University (first experiment) or Ghent University Hospital (UZ-Gent, second experiment) and were paid for their participation. A written informed consent was obtained from all participants.

\section{Distortion Product Otoacoustic Emission}

In the first experiment, distortion product otoacoustic emissions (DPOAEs) were acquired and analyzed using
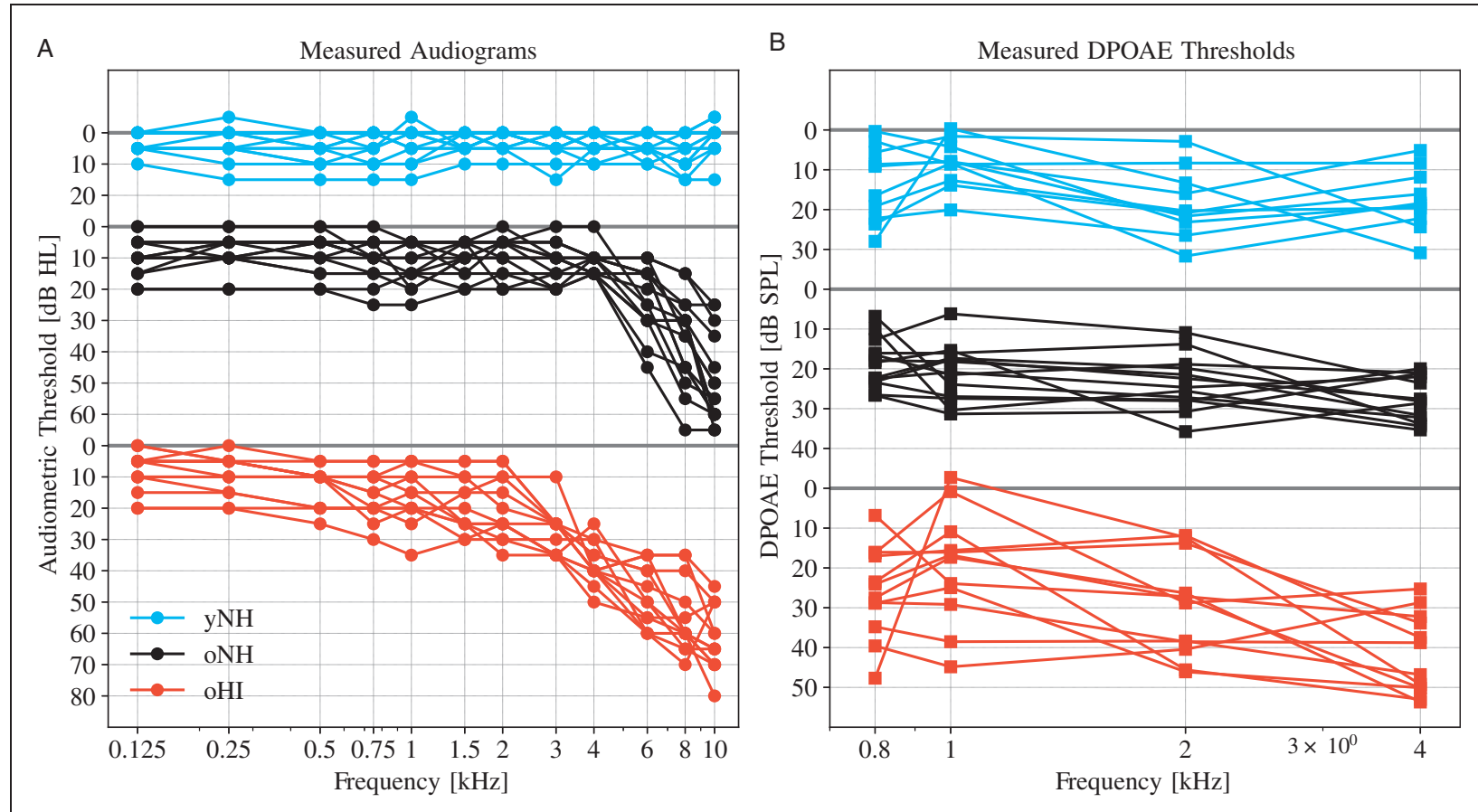

Figure I. Measured audiograms and DPOAE thresholds. A: Audiograms. B: DPOAE thresholds (DPTHs) of the participants in the first experiment.

A: Audiograms. B: DPOAE thresholds (DPTHs) of the participants in the first experiment. DPOAE = distortion-product otoacoustic emission; $y \mathrm{NH}=$ young normal-hearing; oNH = older normal-hearing; oHI = older hearing-impaired. 
a custom-made MATLAB software (Mauermann, 2013). Stimuli were delivered through ER-2 earphones coupled to the ER-10B+ microphone system (Etymotic Research) using a primary frequency sweeping procedure at a fixed $f_{2} / f_{1}$ ratio of 1.2 . The implemented DPOAE paradigm continuously swept the primary frequencies with a rate of $2 \mathrm{~s} /$ octave within a $1 / 3$ octave range around the geometric mean of $\mathrm{f}_{2} \in\{0.8,1,2,4\}$ $\mathrm{kHz}$ (Long et al., 2008). The $\mathrm{L}_{2}$ primary levels ranged between 30 and $60 \mathrm{~dB}-\mathrm{SPL}$ for the $\mathrm{yNH}$ and $\mathrm{oNH}$ groups, using a 6-dB step. The level range was different for the oHI group: 30 to $72 \mathrm{~dB}$-SPL. $\mathrm{L}_{1}$ levels were determined according to the scissors paradigm (Kummer et al., 1998). For a given $f_{2}$ primary frequency, the DP-component $\left(\mathrm{L}_{\mathrm{DP}}\right)$ growth function was plotted as a function of $\mathrm{L}_{2}$ and a cubic curve was fit to the $\mathrm{L}_{\mathrm{DP}}$ data points using a bootstrapping procedure to include the standard deviation of the individual $\mathrm{L}_{\mathrm{DP}}$ data points in the fit (Verhulst et al., 2016). The $\mathrm{L}_{2}$ level at which the cubic curve crossed $-25 \mathrm{~dB}-\mathrm{SPL}$ was determined for each bootstrap average to yield the DPOAE threshold (DPTH) and its standard deviation at a given $\mathrm{f}_{2}$ (Boege \& Janssen, 2002). Derived experimental DPTHs of the $\mathrm{yNH}, \mathrm{oNH}$, and oHI groups are shown in Figure 1B. DPOAEs were not available for the subjects of the validation experiment.

\section{EEG Measurements}

ABR and EFR stimuli were generated in MATLAB and digitized with a sampling rate of $48 \mathrm{kHz}$ for the first data set. Afterwards, they were delivered monaurally through a Fireface UCX external sound card (RME) and a TDTHB7 headphone driver connected to a shielded ER-2 earphone. The electroencephalogram (EEG) signals were recorded with a sampling frequency of $16384 \mathrm{~Hz}$ via a 64-channel Biosemi EEG system using an equidistantly-spaced electrode cap. All active electrodes were placed in the cap using highly conductive gel. The common-mode-sense (CMS) and driven-right-leg (DRL) electrodes were attached to the fronto-central midline and the tip of the nose, respectively. A comprehensive explanation of the experimental configuration can be found in Garrett and Verhulst (2019).

AEPs of the validation experiment were recorded using the SmartEP continuous acquisition module (SEPCAM) of the Universal Smart Box (Intelligent Hearing System, Miami, FL, USA). EFR stimuli were generated in MATLAB using a sampling rate of $20-\mathrm{kHz}$ and stored in a ".wav" format. AEP stimuli were presented monaurally through a shielded ER-2 earphone (Etymotic Research) and AEPs were recorded at a sampling frequency of $10 \mathrm{kHz}$ via Ambu Neuroline 720 snap electrodes connected to vertex, nasion, and both earlobes. The electrodes were placed after a skin preparation procedure using NuPrep gel. The skinelectrode impedance was kept below $3 \mathrm{k} \Omega$ during the recordings.

\section{EFR Stimuli}

We recorded EFRs in response to a 400-ms-long stimuli consisting of a $4-\mathrm{kHz}$ pure-tone carrier and a $120-\mathrm{Hz}$ rectangular-wave modulator with $25 \%$ duty cycle (i.e., the RAM25 in Vasilkov et al., 2021). The stimulus waveform is visualized in the inset of Figure $2 \mathrm{~B}$ and we considered a modulation depth of $95 \%$. Stimuli were presented 1,000 times (500 times in either positive or negative polarity) and had a root-mean-square (RMS) of $68.18 \mathrm{~dB}$-SPL. The calibration of the stimulus was performed to have the same peak-to-peak amplitude as a 70-dB-SPL sinusoidal amplitude modulated (SAM) 4-kHz pure-tone. The $\mathrm{Cz}$ channel recording was re-referenced to the average of the ear-lobe electrodes and 400-ms epochs were extracted relative to the stimulus onset. The mean amplitude of each epoch was subtracted to correct for the baseline-drift. See Vasilkov et al. (2021) for further details on the frequency-domain bootstrapping and noise-floor estimation method. The noise-floor corrected spectral magnitudes $\left(\mathrm{M}_{\mathrm{f}_{\mathrm{k}}}\right)$ at the modulation frequency $\mathrm{f}_{1}=120 \mathrm{~Hz}$ and four harmonics, that is, $\mathrm{f}_{2}$ to $\mathrm{f}_{5}$, were summed up to yield the EFR.

$$
\mathrm{RAM}-\mathrm{EFR}=\sum_{\mathrm{k}=1}^{5} \mathrm{M}_{\mathrm{f}_{\mathrm{k}}}, \quad \mathrm{f}_{\mathrm{k}}=120 \times \mathrm{k}
$$

Figure 2A depicts a typical NH RAM-EFR spectrum and corresponding noise-floor. The arrows show the derived peak-to-noise-floor magnitudes at the modulation frequency and following harmonics. The energy of EFR peak is reduced for the oHI subject shown in the Panel B.

The RAM stimulus in the second experiment (i.e., the validation database) was a $110-\mathrm{Hz} 95 \%$ modulated $4-\mathrm{kHz}$ pure tone. The $500-\mathrm{ms}$ stimulus was presented 1,000 times with alternating polarity (500 each) and had a $70 \mathrm{~dB}-S P L$ level. The acquired AEPs were initially saved in ".EEG.F" format on SEPCAM and were afterwards converted to ".mat" format using the custom-made "sep-cam2mat" MATLAB function for offline analysis. EFRs recorded from the vertex electrode were rereferenced to the ipsilateral earlobe electrode and filtered between 30 and $1500 \mathrm{~Hz}$ using an 800th order Blackman-window based finite-impulseresponse (FIR) filter. Epoching was applied to the steady state part of the response, that is, 100 to $500 \mathrm{~ms}$ of the response relative to the stimulus onset. The baseline drift was corrected by subtracting the mean of each epoch, afterwards 200 epochs with the highest 


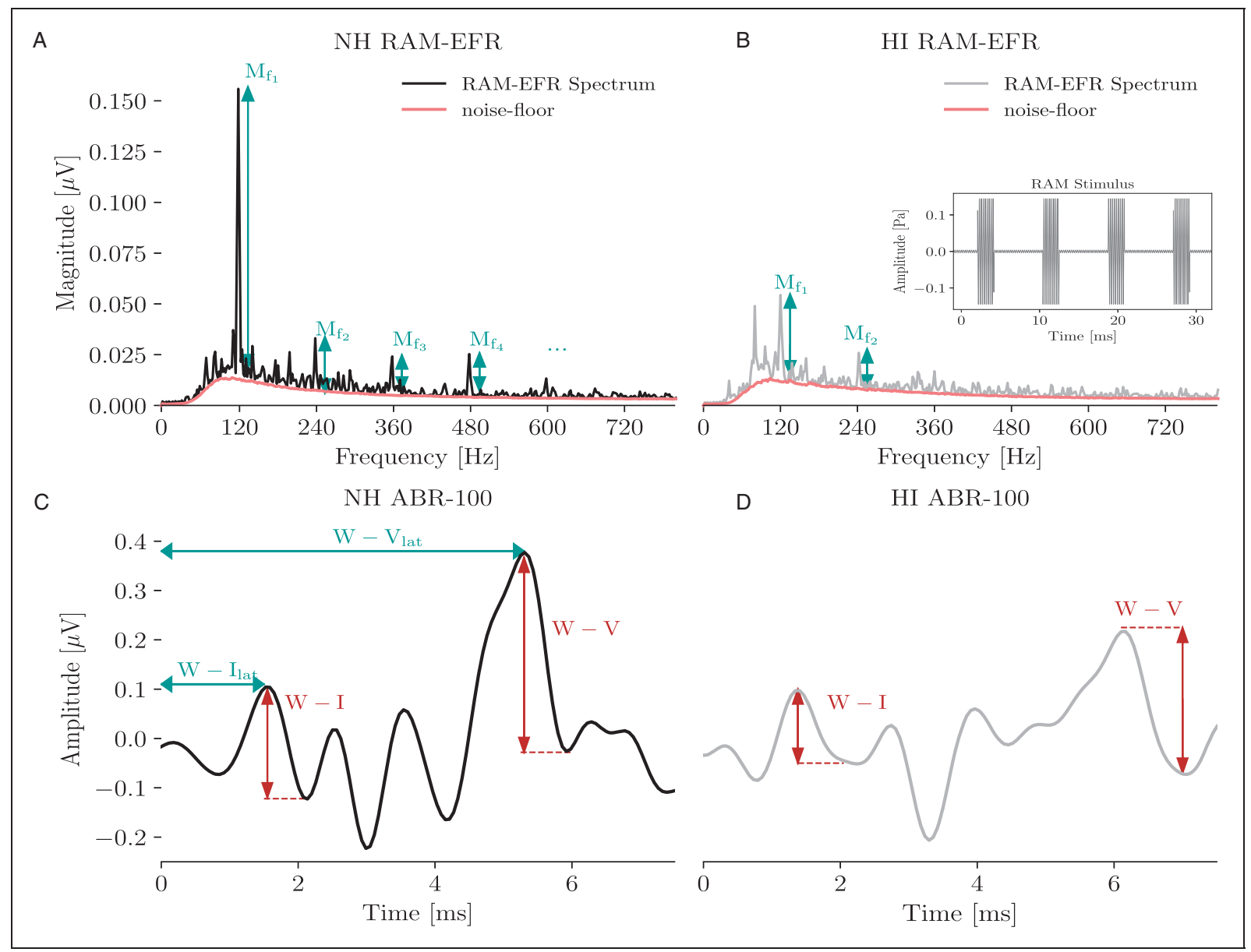

Figure 2. Comparison of Exemplary NH and HI RAM-EFRs and ABRs. A: RAM-EFR of a yNH subject (yNHI5) and the corresponding noise-floor (NF). Arrows specified by $M_{f}$ show the peak-to-noise floor magnitudes at the modulation frequency, that is, $120 \mathrm{~Hz}$ and the following harmonics. B: RAM-EFR of an oHI subject (oHI) and the corresponding NF. C: ABR of a yNH subject (yNHI5). Arrows show the extracted wave-I and $\mathrm{V}$ amplitudes and latencies. $\mathrm{D}$ : $\mathrm{ABR}$ of an oHI subject (oHII2). $\mathrm{NH}=$ normal hearing; EFR = envelope-following response; $\mathrm{HI}=$ hearing-impaired; $\mathrm{ABR}=$ auditory brainstem response.

peak-to-trough values were rejected. The amplitudes of the remained epochs did not exceed $100 \mu \mathrm{V}$. A frequency-domain bootstrapping approach was adopted to estimate the noise-floor and to remove it from the averaged trials using the method proposed in $\mathrm{Zhu}$ et al. (2013). To this end, we calculated the fast Fourier Transform (FFT) of 800 epochs to generate 400 mean spectra by randomly sampling the 800 epochs with replacement (keeping an equal number of polarities in the draw). Averaging the resampled spectra yielded the $i$-th mean-EFR spectrum $\left(\mathrm{EFR}_{\mathrm{raw}_{\mathrm{i}}}\right)$

$$
\mathrm{EFR}_{\mathrm{raw}_{\mathrm{i}}}=\frac{2}{\mathrm{n}}\left|\mathrm{X}_{\mathrm{i}}\right|, \quad \mathrm{i}=1, \ldots, 400
$$

where $\mathrm{X}_{\mathrm{i}}$ stands for the $i$-th averaged resampled spectra and $\mathrm{n}$ is the number of FFT points $(n=10,000)$. To calculate the spectral noise floor, we repeated the resampling procedure 1,500 times, but used phaseflipped odd epochs

$$
\mathrm{NF}_{\mathrm{j}}=\frac{2}{\mathrm{n}}\left|\mathrm{Y}_{\mathrm{j}}\right|, \quad \mathrm{j}=1, \ldots, 1500
$$

In Equation 3, $\mathrm{Y}_{\mathrm{j}}$ is the $j$-th averaged resampled spectra with phase-flipped odd epochs. Finally, we subtracted the NF mean $(\overline{\mathrm{NF}})$, from each of the 400 bootstrapped mean-EFRs $\left(\mathrm{EFR}_{\mathrm{raw}_{\mathrm{i}}}\right)$ to derive $400 \mathrm{NF}$ corrected EFR spectra

$$
\mathrm{EFR}_{\mathrm{Spec}_{\mathrm{i}}}=\mathrm{EFR}_{\mathrm{raw}_{\mathrm{i}}}-\overline{\mathrm{NF}}, \quad \mathrm{i}=1,2, \ldots, 400
$$

The peaks of the EFR $\mathrm{raw}_{\mathrm{i}}$ at the modulation frequency of stimulus $\left(\mathrm{f}_{1}=110 \mathrm{~Hz}\right)$, and the next four harmonics were identified if they were above the $\overline{\mathrm{NF}}$. We defined the RAM-EFR $\mathrm{R}_{\mathrm{i}}$ by summing the magnitudes of the 
identified peaks for each $E R_{\text {Spec }_{i}}$. The RAM-EFR metric mean and variability was defined by the mean and standard deviation of RAM-EFR $\mathrm{R}_{\mathrm{i}}$ over 400 samples.

Auditory Brainstem Responses. ABRs were recorded to 80$\mu s-l o n g$ alternating polarity clicks presented at 70 and $100 \mathrm{~dB}$-peSPL. Stimuli were presented through the setup explained in Garrett et al. (2019) and repeated 3,000 times with a rate of $10 \mathrm{~Hz}$ using a uniformly distributed random interstimulus interval of $100 \mathrm{~ms} \pm$ $10 \mathrm{~ms}$. Cz-channel recordings were rereferenced to the contralateral earlobe electrode and filtered between 100 and $1500 \mathrm{~Hz}$. Moreover, $25 \mathrm{~ms}-1$ ong epochs, that is, -5 to 20-ms relative to the stimulus onset, were extracted and corresponding mean values were subtracted to perform a baseline correction. Then, each positive polarity epoch was averaged with the following negative epoch and 100 paired-averages with the highest peak-to-trough values were rejected. The remaining pair-averaged epochs had amplitudes below $25 \mu \mathrm{V}$. To include ABR variability in our analysis and to estimate the ABR noise floor, we applied the bootstrapping approach of $\mathrm{Zhu}$ et al. (2013), in the time domain. In addition, 2,000 and 4,500 epochs were drawn for the signal and noisefloor estimation, respectively. Half of the noise-floorestimation epochs (i.e., 2,250 pair-averaged drawn epochs with replacement) were multiplied by -1 before final averaging. Finally, the estimated noise-floor mean was subtracted from the 2,000 averaged epochs to yield mean noie-floor-corrected ABR waveforms. ABR wave$\mathrm{I}$ and $\mathrm{-V}$ peak and trough amplitudes and corresponding latencies were determined by visual inspection from the mean ABR waveform and were confirmed by an audiologist. Figure 2 (Panels $\mathrm{C}$ and D) compares ABR waveforms of a $y \mathrm{NH}$ and $\mathrm{oHI}$ subject from the cohort and indicates the identified ABR peaks and latencies. To extract peak latencies and amplitudes from the bootstrapped data, wave maxima and minima were detected in $1,1.8,0.5$, and $1.5 \mathrm{~ms}$ intervals around the wave- $\mathrm{I}_{70}$, wave- $\mathrm{V}_{70}$, wave- $\mathrm{I}_{100}$, wave- $\mathrm{V}_{100}$ peaks and troughs, identified from the mean ABR waveform. The interval ranges were determined based on visual inspection. ABR wave-I and V latencies were shifted by $1.16 \mathrm{~ms}$ to compensate for the delay introduced by the sound delivery system.

We used a total of 13 ABR and EFR markers in the development phase and one EFR marker in the validation phase. Table 1 details the definition of each metric and lists the corresponding abbreviations used in this paper. The last column defines the variability metric associated with each marker, which were obtained from the earlier described bootstrapping procedure. To determine the measurement variability of ABR growth-slopes, we applied error propagation to account for the standard deviations of two different measures from the same listener, for example, ABR-70 and ABR-100. In this case, the bootstrapped metrics were drawn from the $95 \%$ confidence interval of a normal distribution characterized by the mean of the metric and its bootstrapped standard deviation. The bootstrapping technique described in this section, provided a tool to estimate the variability of AEP-derived metrics and to incorporate them in the proposed classification approach. Obtained standard deviations from bootstrapping can be used to measure the CS-profiling prediction robustness of the study participants.

Table I. Extracted AEP-Metrics Definitions and Corresponding Standard Deviations.

\begin{tabular}{|c|c|c|c|}
\hline Metric & Symbol & Definition & Measure of variability \\
\hline Rectangular-wave & RAM-EFR & Equation I & $\sigma_{\text {boot }(\text { RAM-EFR })}$ \\
\hline \multicolumn{4}{|l|}{ Amplitude-modulated EFR } \\
\hline ABR-70 wave-I amplitude & $w-I_{70}$ & $w-I_{70(\text { peak })}-w-I_{70(\text { trough-after })}$ & \\
\hline ABR-I00 wave-I amplitude & $w-I_{100}$ & $\mathrm{~W}-\mathrm{I}_{100(\text { peak) }}-\mathrm{W}-\mathrm{I}_{100(\text { trough-after) }}$ & $\sigma_{\text {boot(peak-to-trough) }}$ \\
\hline ABR-70 wave- $V$ amplitude & $w-V_{70}$ & $w-V_{70(\text { peak })}-w-V_{70 \text { (trough-after) }}$ & \\
\hline ABR-I00 wave-V amplitude & $w-V_{100}$ & $w-V_{100 \text { (peak) }}-w-V_{100(\text { trough-after) }}$ & \\
\hline ABR-70 wave-I latency & $w-I_{\text {lat70 }}$ & $\mathrm{w}-\mathrm{I}_{70(\text { peak) }}$ latency & \\
\hline ABR-I00 wave-I latency & $w-I_{\text {lat } 100}$ & $w-I_{100(\text { peak) }}$ latency & $\sigma_{\text {boot(latency) }}$ \\
\hline ABR-70 wave- $V$ latency & $w-V_{\text {lat70 }}$ & $w-V_{70 \text { (peak) }}$ latency & \\
\hline ABR-I00 wave-V latency & $w-V_{\text {lat } 100}$ & $w-V_{100 \text { (peak) latency }}$ & \\
\hline ABR wave-I amplitude growth & w-I-growth & $\frac{w-I_{100}-w-I_{70}}{100-70}$ & $\frac{\mathrm{I}}{\mathrm{N}} \sqrt{\sigma_{\text {boot }\left(\mathrm{w}-\mathrm{I}_{100}\right)}^{2}+\sigma_{\text {boot }\left(\mathrm{w}-\mathrm{I}_{70}\right)}^{2}}$ \\
\hline ABR wave- $V$ amplitude growth & w-V-growth & $\frac{w-V_{100}-w-V_{70}}{100-70}$ & $\frac{1}{\mathrm{~N}} \sqrt{\sigma_{\text {boot }\left(\mathrm{w}-\mathrm{V}_{100}\right)}^{2}+\sigma_{\text {boot }\left(\mathrm{w}-\mathrm{V}_{70}\right)}^{2}}$ \\
\hline ABR wave-I latency growth & $w-I_{\text {lat }}-$ growth & $\frac{\left|w-I_{\text {lat } 100}-w-I_{\text {lat70 }}\right|}{100-70}$ & $\frac{1}{N} \sqrt{\sigma_{\text {boot }\left(w-I_{\text {lat } 100}\right)}^{2}+\sigma_{\text {boot }\left(w-I_{\text {lat } 70}\right)}^{2}}$ \\
\hline ABR wave-V latency growth & $w-V_{\text {lat }}-$ growth & $\frac{\left|w-V_{\text {lat } 100}-w-V_{\text {lat70 }}\right|}{100-70}$ & $\frac{1}{\mathrm{~N}} \sqrt{\sigma_{\text {boot }\left(\mathrm{w}-\mathrm{V}_{\text {lat } 100)}\right)}+\sigma_{\text {boot }\left(\mathrm{w}-\mathrm{V}_{\text {lat } 70}\right)}^{2}}$ \\
\hline
\end{tabular}

Note. $\mathrm{EFR}=$ envelope-following response; $\mathrm{ABR}=$ auditory brainstem response. In the last column, $\sigma$ represents the standard deviation. $\sigma_{\text {boot }}$ is the standard deviation of the bootstrapped metric. 


\section{Individualized Auditory Periphery Model}

To simulate individualized SNHL profiles that would match the histopathology of the study participants, we used a computational model of the auditory periphery (Osses \& Verhulst, 2019; Verhulst et al., 2018). In the first step, we personalized the cochlear model parameters on the basis of OHC markers of SNHL (audiogram or DPTH). Afterwards, we simulated AEPs for different degrees of CS and compared the simulations to the recordings to develop and test our auditory profiling method. Figure 3 schematizes the auditory model individualization.

\section{Cochlear Model Individualization}

Measured audiograms and DPTHs were used independently to determine the individual CGL parameters (in $\mathrm{dB}-\mathrm{HL}$ ) of the cochlear transmission-line (TL) model, shown in pink in Figure 3. In our approach, CGL determines the double pole of the cochlear admittance through the gain and tuning of the cochlear filters (Verhulst et al., 2012). We thus model the consequence of $\mathrm{OHC}$ damage or presbycusis without specifically accounting for damage of the stereocilia or sensory cells.
From here on, mAudTH and sAudTH refer to measured and simulated audiometric thresholds, respectively. Likewise, mDPTH and sDPTH stand for measured and simulated DPOAE thresholds.

Audiogram-Based Cochlear Filter Pole-Setting. Here, we translated the frequency-specific audiometric dB-HL (Figure 1A) into cochlear filter gain loss. These values were translated into double-pole values of the cochlear admittance function across CF (see Verhulst et al., 2016).

Specifically, at a CF corresponding to a measured audiometric frequency $\left(\mathrm{CF}=\mathrm{f}_{\text {aud }}\right)$, the power spectrum of the $\mathrm{NH}$ basilar membrane (BM) impulse response, $\mathrm{H}_{\mathrm{NH}}\left(\mathrm{f}_{\text {aud }}\right)$, served as reference before the gain loss was applied. Among a range of cochlear filter pole-values in $[0.036,0.302]$, the pole value, $\alpha_{\mathrm{A}}^{*}\left(\mathrm{f}_{\mathrm{aud}}\right)$, that causes a relative gain-loss equal to $\operatorname{mAudTH}\left(\mathrm{f}_{\text {aud }}\right)$, was assigned. Thereby, the $\mathrm{CGL}$ at $\mathrm{CF}=\mathrm{f}_{\text {aud }}$ is given by

$$
\operatorname{CGL}\left(\mathrm{f}_{\text {aud }}\right)=\mathrm{H}_{\mathrm{NH}}\left(\mathrm{f}_{\text {aud }}\right)-\mathrm{H}_{\alpha_{\mathrm{A}}^{*}}\left(\mathrm{f}_{\text {aud }}\right)
$$

where $H_{\alpha_{A}^{*}}\left(f_{\text {aud }}\right)$ equals the power spectrum of the BM impulse response at $\mathrm{CF}=\mathrm{f}_{\text {aud }}$ with a pole value of $\alpha_{\mathrm{A}}^{*}$ that causes a CGL equal to $\mathrm{mAudTH}\left(\mathrm{f}_{\text {aud }}\right)$. This procedure was repeated for all CF channels corresponding to

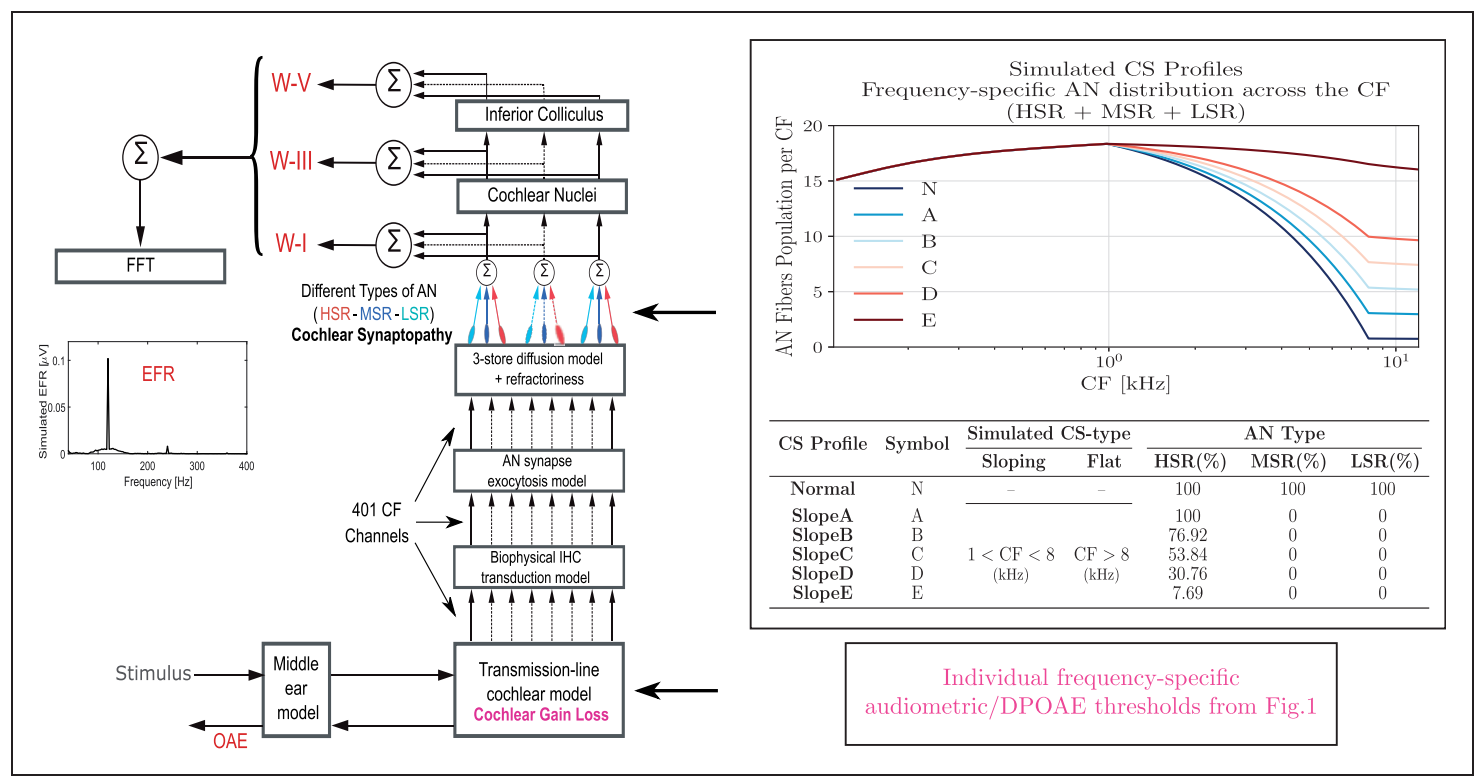

Figure 3. Auditory Model Individualization. The block diagram on the left depicts the different stages of the employed auditory periphery model (Verhulst et al., 2018). Experimentally measured audiometric thresholds were inserted to the transmission-line cochlear model to adjust BM admittance function poles. The box on top-right corner shows the nonuniform AN population distribution across the CF for simulated different degrees of CS profiles. The profile without CS is shown in dark brown (indicated with N) and higher degrees of CS are shown according to the color map. HSR = high-spontaneous-rate; MSR = medium-spontaneous-rate; LSR = low-spontaneous-rate; $\mathrm{EFR}=$ envelope-following response; $\mathrm{FFT}=$ fast Fourier Transform; CS = cochlear synaptopathy; $\mathrm{AN}=$ auditory nerve; $\mathrm{DPOAE}=$ distortion-product otoacoustic emission. 
measured audiometric frequencies and individualized cochlear filter pole-functions were obtained by interpolating the pole values across CF (Verhulst et al., 2016).

We employed the predicted pole functions to simulate individual audiograms and to evaluate the prediction error. To this end, individualized AN excitation patterns (ANEP) were simulated in response to 500-ms pure tones presented at audiogram frequencies $\left(\mathrm{f}_{\text {aud }}\right)$ using 62 intensity levels (L) between -5 and $55 \mathrm{~dB}$-SPL. We defined ANEP as the RMS of the AN firing rate at each $\mathrm{CF} \in \mathrm{f}_{\text {aud }}$ and determined on-CF peaks of the presented level series as $\operatorname{ANEP}_{\left(\mathrm{f}_{\text {aud }}, \mathrm{L}\right)}$. We simulated NH ANEPs using $\mathrm{NH}$ pole-function at the threshold of audibility in a frequency-specific manner $\left(\mathrm{L}_{\mathrm{NH}}\left(\mathrm{f}_{\mathrm{aud}}\right)\right)$, that is, the zero-phon curve of the equal-loudness-contour (ISO 226:1987). From this reference NH curve, we calculated the simulated audiometric thresholds (sAudTH) of the experiment participants as follows

$$
\mathrm{L}_{\min }\left(\mathrm{f}_{\text {aud }}\right)=\underset{\mathrm{L} \in[-5,55]}{\arg \min }\left[\operatorname{ANEP}_{\left(\mathrm{f}_{\text {aud }}, \mathrm{L}_{\mathrm{NH}}\left(\mathrm{f}_{\text {aud }}\right)\right)}-\operatorname{ANEP}_{\left(\mathrm{f}_{\text {aud }}, \mathrm{L}\right)}\right]
$$

$$
\operatorname{sAudTH}\left(\mathrm{f}_{\text {aud }}\right)=\mathrm{L}_{\min }\left(\mathrm{f}_{\mathrm{aud}}\right)-\mathrm{L}_{\mathrm{NH}}\left(\mathrm{f}_{\mathrm{aud}}\right)
$$

Figure 4A shows grand-averaged mAudTHs and sAudTHs across the $\mathrm{yNH}$, oNH, and oHI groups. In addition, Figure 4C compares the sAudTH (dashed lines) and mAudTH (solid lines) of an example yNH and oHI subject. Note that simulating CGLs greater than $35 \mathrm{~dB}-\mathrm{HL}$ is impossible in our cochlear model, which has a maximal applicable cochlear mechanical gain of $35 \mathrm{~dB}$. In the last step, we estimated the absolute prediction error as follows

$$
\operatorname{err}_{\text {audio }}\left(f_{\text {audio }}\right)=\left|\operatorname{mAudTH}\left(\mathrm{f}_{\text {aud }}\right)-\operatorname{sAudTH}\left(\mathrm{f}_{\text {aud }}\right)\right|
$$

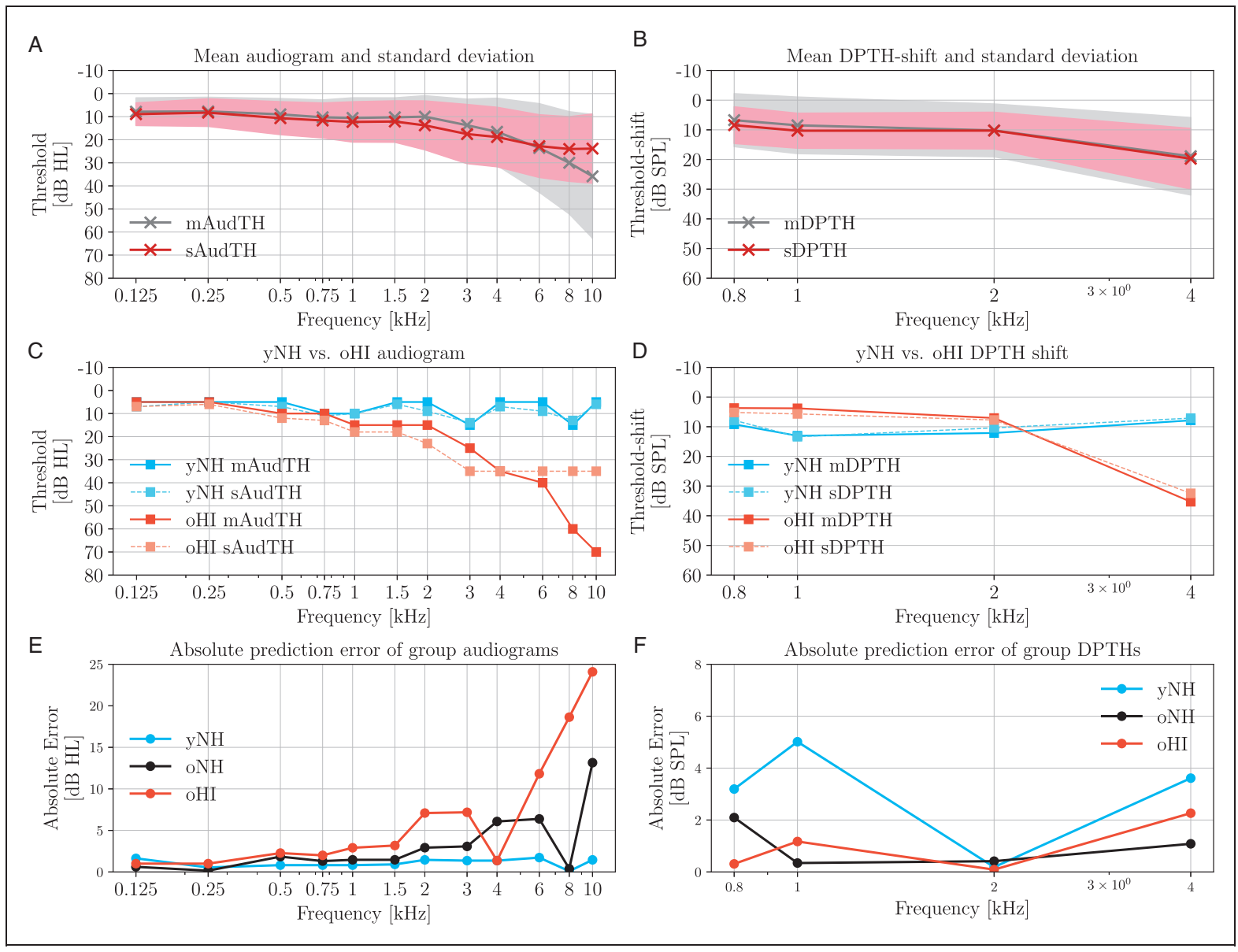

Figure 4. A Comparison Between the Measured and Simulated AudTHs and DPTHs. The average (solid) and standard deviation (shaded area) of the measured (gray) and simulated (red) AudTHs and DPTHs are shown in Panels A and B, respectively. A comparison between sAudTH and mAudTH of a yNH and oHI listener is shown in Panel C. Panel D compares the sDPTH (dotted) and mDPTH (solid) of the same $y \mathrm{NH}$ and oHI listeners (Panel C). Frequency-specific group-averaged absolute prediction errors of AudTH and DPTH are shown in Panels $\mathrm{E}$ and $\mathrm{F}$, respectively (yNH: blue, oNH: black, oHI: orange). DPTH $=\mathrm{DPOAE}$ threshold; $y \mathrm{NH}=$ young normal-hearing; oNH $=$ older normal-hearing; $\mathrm{oHI}=$ older hearing-impaired. 
Figure 4E compares the mean absolute errors on a group-level basis. The elevated error of the oHI group at high frequencies is due to the model limitation in simulating gain losses greater than $35 \mathrm{~dB}-\mathrm{HL}$.

DPTH-Based Cochlear Filter Pole Setting. Implementing DPTH-based cochlear model individualization was complicated by the fewer DPTHs we had available, that is, four frequencies, compared to 12 AudTHs. Hence, a simple interpolation to determine poles between the measured frequencies, yielded large prediction errors. In addition, the longitudinal filter coupling and associated gain propagation along the cochlear partition complicated matters. To tackle these issues, we trained a machine-learning algorithm to map DPTHs via cochlear travelling waves to corresponding cochlear filter pole functions across CF. Once trained, we need only a few measured DPTHs to make a relatively accurate prediction of individual pole values. Figure 5 illustrates the complete procedure.
First, we constructed the training data (Figure 5A) using 26 sets of random cochlear filter pole functions. Each set contained $1001 \mathrm{CF}-d e p e n d e n t$ poles and random pole values lay between 0.036 and 0.302 , covering the pole values associated with both $\mathrm{NH}$ and $\mathrm{HI}$ profiles. In addition, three reference pole functions were included as part of the training: $\mathrm{NH}_{\text {poles }}(\mathrm{NH}$ poles), flat ${ }_{\text {min }}$ with across-CF poles of 0.036 (maximally intact cochlea) and flat ${ }_{\max }$, with across-CF poles of 0.302 ( $35 \mathrm{~dB}-\mathrm{HL}$ across $\mathrm{CF}$ ). We employed the generated pole functions and simulated DP amplitudes $\left(\mathrm{sL}_{\mathrm{DP}}\right.$ : the magnitude of $2 \mathrm{f}_{1}-\mathrm{f}_{2}$ ) to train the mapping function. The considered $\mathrm{f}_{2}$ primary frequencies, that is, $0.8,1,2$, and $4 \mathrm{kHz}\left(\mathrm{f}_{1}=\mathrm{f}_{2} / 1.2\right)$ corresponded to the recordings we had available and $\mathrm{L}_{2}$ levels $(-10$ to $70 \mathrm{~dB}-\mathrm{SPL}$, with a step of 5-dB). We simulated DPOAE inputoutput ( $\mathrm{I} / \mathrm{O})$ functions at each $\mathrm{f}_{2}$ frequency and determined the SDPTH as the $\mathrm{L}_{2}$ level at which the $s L_{D P}$ growth function crossed the $\mathrm{L}_{2}$ of $-10 \mathrm{~dB}-\mathrm{SPL}$. We chose a $-10 \mathrm{~dB}$-SPL threshold for our simulations,

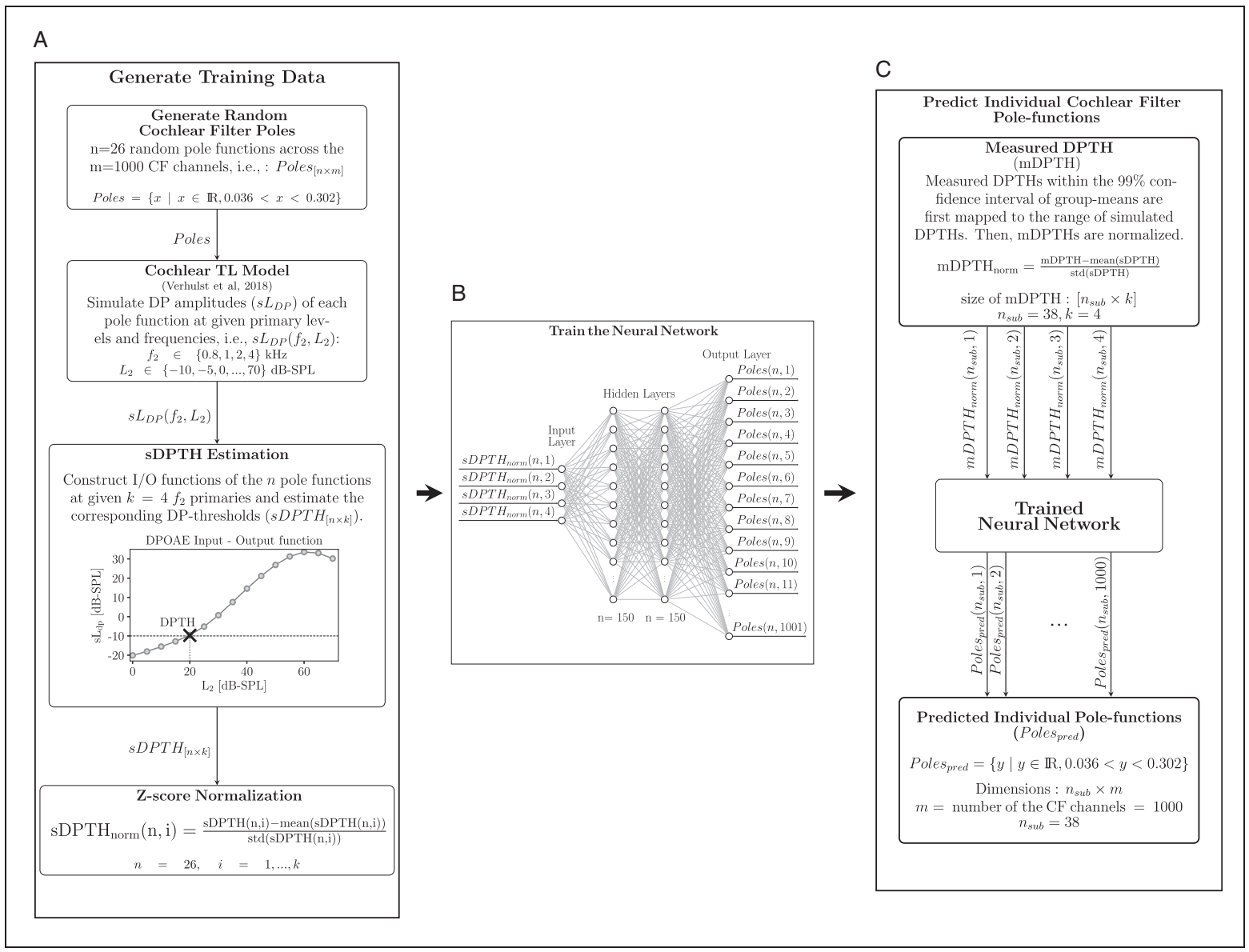

Figure 5. Neural Network-Based Cochlear Model Individualization Using Measured and Simulated DPTHs. A: Random cochlear filter

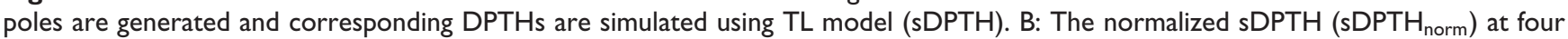
frequencies are introduced to the neural network as input. The random pole values generated in (A) are served as training target for sDPTH norm. $_{\text {. }}$ : Measured DPTHs (mDPTHs) are fed into the trained neural network after pre-processing and individualized cochlear filter pole functions are predicted. DPTH = DPOAE threshold; DPOAE = distortion-product otoacoustic emission. 
given that the conventional experimental $-25 \mathrm{~dB}-\mathrm{SPL}$ crossing point yielded inconclusive sDPTH, in particular for pole values associated with greater CGLs. sDPTH values for 26 sets of pole-functions at four primary frequencies were fed into the neural network after normalization $\left(\mathrm{sDPTH}_{\text {norm }}\right.$, Figure $\left.5 \mathrm{~B}\right)$ to train it to map frequency-specific $\mathrm{sDPTH}_{\text {norm }}$ values (input) to $\mathrm{CF}$ dependent pole functions (output).

The architecture of the designed neural network is shown in Figure 5B and consists of an input layer of four neurons, two hidden layers of 150 neurons and an output layer of 1001 neurons. A standard sigmoid activation function (i.e., between 0 and 1 ) was applied to the hidden layers. A customized sigmoid activation function (between 0.036 and 0.302) was employed in the output layer to yield the desired range of the cochlear model pole functions. An ADAM optimizer with a learning rate of 0.001 was applied to minimize the meansquare-error (MSE) of the learning algorithm. The method was developed in Python using Keras library and Tensorflow backend.

The trained neural network was employed to predict individualized pole functions given DPTHs of the experimental cohort (Figure 5C). Prior to the prediction, mDPTHs needed to be preprocessed to determine a suitable experimental range of DPTHs for the mapping. Among the 41 subjects, six subjects (yNH: three, oNH: two, and oHI: one) without complete mDPTH values at all measured frequencies were dropped. In each of the three recruited groups, the $99 \%$ confidence interval around the frequency-specific group means were specified and $\mathrm{mDPTH}$ values that either exceeded or fell below of those intervals were set to extremum values. Then, mDPTHs were mapped to the range of the sDPTH associated with reference flat $t_{\min }\left(\mathrm{sDPTH}_{\mathrm{flat}_{\text {min }}}\right)$ and flat $_{\text {max }}\left(\mathrm{sDPTH}_{\mathrm{flat}_{\max }}\right)$ pole functions. Afterwards, mapped mDPTHs $\left(\mathrm{mDPTH}_{\mathrm{map}}\right)$ were normalized $\left(\mathrm{mDPTH}_{\text {norm }}\right)$ and given to the trained neural network to predict personalized pole-functions. To assess the prediction error, the predicted pole functions (Poles $_{\text {pred }}$ in Figure 5C), were used to simulate individualized sDPTHs that were compared to the individual mDPTHs $f_{2}$ primary frequencies. mDPTHs and sDPTHs were referenced to the simulated DPTHs of a model with $\mathrm{NH}_{\text {poles }}$ as follows

$$
\begin{gathered}
\operatorname{sDPTH}_{\text {ref }}\left(f_{2}\right)=\operatorname{sDPTH}\left(f_{2}\right)-\operatorname{sDPTH}_{\mathrm{NH}}\left(\mathrm{f}_{2}\right) \\
\operatorname{mDPTH}_{\text {ref }}\left(\mathrm{f}_{2}\right)=\operatorname{mDPTH}_{\text {map }}\left(\mathrm{f}_{2}\right)-\operatorname{sDPTH}_{\mathrm{NH}}\left(\mathrm{f}_{2}\right)
\end{gathered}
$$

$\mathrm{sDPTH}_{\mathrm{NH}}\left(\mathrm{f}_{2}\right)$ refers to the frequency-specific sDPTH values simulated using the model with $\mathrm{NH}_{\text {poles }}$. Obtained $\mathrm{sDPTH}_{\text {ref }}$ and $\mathrm{mDPTH}_{\text {ref }}$ from Equations 9 and 10 were mapped back to the experimental range according to
Equations 11 and 12, and corresponding grandaverages and standard deviations are shown in Figure 4B. More specifically, Figure 4C compares measured and simulated DPTH-shifts for a yNH and oHI subject.

$$
\begin{aligned}
& \mathrm{sDPTH}_{\text {shift }}\left(\mathrm{f}_{2}\right)=\mathrm{sDPTH}_{\text {ref }}\left(\mathrm{f}_{2}\right) \\
& \frac{\max \left[\operatorname{mDPTH}\left(\mathrm{f}_{2}\right)\right]-\min \left[\operatorname{mDPTH}\left(\mathrm{f}_{2}\right)\right]}{\text { sDPTH }_{\text {flat }_{\text {max }}}\left(\mathrm{f}_{2}\right)-\mathrm{sDPTH}_{\text {flat }_{\text {min }}}\left(\mathrm{f}_{2}\right)} \\
& \mathrm{mDPTH}_{\text {shift }}\left(\mathrm{f}_{2}\right)=\mathrm{mDPTH}_{\text {ref }}\left(\mathrm{f}_{2}\right) \\
& \frac{\left.\max \left[\operatorname{mDPTH}\left(\mathrm{f}_{2}\right)\right)\right]-\min \left[\operatorname{mDPTH}\left(\mathrm{f}_{2}\right)\right]}{\operatorname{sDPTH}_{\mathrm{flat}_{\text {max }}}\left(\mathrm{f}_{2}\right)-\mathrm{sDPTH}_{\mathrm{flat}_{\text {min }}}\left(\mathrm{f}_{2}\right)}
\end{aligned}
$$

Finally, the prediction error was calculated as in Equation 13, and the absolute mean error for each group is shown in Figure 4F.

$$
\operatorname{err}_{\text {dpth }}\left(f_{2}\right)=\left|\mathrm{mDPTH}_{\text {shift }}\left(\mathrm{f}_{2}\right)-\mathrm{sDPTH}_{\text {shift }}\left(\mathrm{f}_{2}\right)\right|
$$

The developed machine-learning approach can be used to personalize cochlear model parameters based on an objective measure of OHC damage (DPTH) and predict individual CS profiles. CS profiling can be compared for either the DPTH or AudTH-based cochlear model individualization method, and when no DPTHs are available, the standard audiogram-based method can be adopted.

\section{Simulating CS Profiles}

We employed the AudTH- and DPTH-based individualized CGL models to simulate EFRs and ABRs for different CS profiles. To introduce CS, the simulated normal-hearing AN fiber populations, the $N$ CS profile in Figure 3, was reduced in a CF-specific manner. Five additional CS profiles were simulated by proportionally lowering the number of different AN types, starting from low- and medium-spontaneous-rate (LSR and MSR) fibers in profile $A$ to the most severe AN loss in $E$ that only kept $7.69 \%$ of the high-spontaneous-rate (HSR) fiber population. The table in Figure 3 details the AN fiber numbers and types considered for each of the six simulated CS profiles. IHC-related dysfunctions were not considered in this study, given that low degrees of CS do not cause IHC damage (Furman et al., 2013; Kujawa \& Liberman, 2009; Shaheen et al., 2015). However, removing all AN fibers from an IHC in the model would functionally correspond to IHC damage. The CF dependence of the AN population was considered in two steps: (a) Following the CF-dependent AN distribution observed in rhesus monkey (Keshishzadeh et al., 2020; Valero et al., 2017), we applied a nonuniform $\mathrm{NH}$ AN fiber population. (b) CF-specific ANdamage profiles were simulated. The former was 
achieved by mapping the counted CF-dependent AN fibers population in the rhesus monkey (Valero et al., 2017) to the human cochlea, using a distribution of $\mathrm{N}_{\mathrm{HSR}}=68 \%, \mathrm{~N}_{\mathrm{MSR}}=16 \%$ and $\mathrm{N}_{\mathrm{LSR}}=16 \%$ at each CF (Liberman, 1978). Then, sloping high-frequency AN-fiber loss was applied across CF with the assumption that CS starts from the higher frequencies first $(\mathrm{Wu}$ et al., 2020). We ran EFR/ABR simulations for different AN fiber damage profiles, which were characterized by a sloping loss of between 1 and $8 \mathrm{kHz}$. Above $8 \mathrm{kHz}$, we applied a frequency-independent loss.

For every subject we simulated AEPs for each CS profile, after we personalized the cochlear models using either the AudTH-or DPTH-based method. The stimuli adopted for these simulations were identical to those adopted experimentally, but were digitized using a sampling rate of $100 \mathrm{kHz}$, rather than $48 \mathrm{kHz}$. Simulated instantaneous firing rates from the $\mathrm{AN}$, cochlear nucleus $(\mathrm{CN})$, and IC model stages, namely, ABR wave-I, III, and $\mathrm{V}$, respectively, were added up to simulate EFRs (Figure 3). RAM-EFR magnitudes were calculated using Equation 1.

To simulate ABRs, $80-\mu$ s clicks were presented to the model with a continuous sequence of 50 repetitions of alternating polarities (100 in total) and a rate of $10 \mathrm{~Hz}$. Sequential stimulus presentation was adopted to account for the adaptation properties of AN fibers. Individual ABR wave-I and $V$ latencies and amplitudes were extracted by averaging the peak-to-trough values of the response to the last, that is, 50th, positive and negative clicks. The simulated ABR wave-I and V latencies were, respectively, shifted by 1 and $3 \mathrm{~ms}$ to match latencies of recorded ABRs. These values were determined to match the measured yNH group-mean ABR wave-I and $\mathrm{V}$ latencies (at $100 \mathrm{~dB}$-peSPL) with the grand-average individualized ABR simulations across the $\mathrm{yNH}$ group. Given that simulated ABR latencies were not impacted by CS, the applied latency shifts will not confound the CS prediction.

\section{Individual Synaptopathy Profile Predictions}

In previous sections, cochlear model parameters of the subjects were determined using either AudTH- or DPTH-based methods and 13 personalized AEPderived metrics were simulated for six CS profiles of each experiment participant. Here, we develop a classification approach, forward-backward classification, to predict the simulated CS profile that best matches recorded individual AEP metrics and determine the AEP metric that gives the most accurate segregation of simulated individualized CS profiles. This step was implemented separately for either of the cochlear individualization methods. After excluding eight subjects from the cohort (six without complete DPTHs and two with undetectable ABRs), we developed our individual SNHL-profiling method on data from 35 subjects (yNH: 12, oNH: 12, and oHI: 11).

Before classification, we first normalized the 13 AEP metrics (Table 1) derived from measured (M) and simulated six CS profiles per individual (S). The normalized S and $\mathrm{M}$ were calculated using Equations 14 and 15.

$$
\mathrm{S}_{\mathrm{norm}}=\frac{\mathrm{S}-\bar{S}}{\sigma_{\mathrm{S}}}
$$

$\mathrm{S}$ is the matrix of simulated AEP metrics and contains 210 rows ( 35 subjects with 6 CS profiles) and 13 columns, the number of derived AEP metrics. $\bar{S}$ and $\sigma_{\mathrm{S}}$ refer to the mean and standard deviation of S, respectively.

$$
\mathrm{M}_{\text {norm }}=\frac{\mathrm{M}-\bar{S}}{\sigma_{\mathrm{S}}}
$$

In Equation 15, $\mathrm{M}$ refers to the matrix of measured AEP metrics with a dimension of $35 \times 13$. We created 8,191 feature-sets using all possible combinations of 13 metrics $\left(\sum_{i=1}^{13}\left(\begin{array}{c}13 \\ i\end{array}\right)=8191\right)$. Metrics combination was performed separately for $\mathbf{M}_{\text {norm }}$ and $\mathrm{S}_{\text {norm }}$. The number of metrics in each feature set varied between one and 13 . From here on, $\mathbf{F}$ refers to the constructed 8,191 feature sets of AEP-derived metrics and $\mathrm{F}_{\mathrm{i}}$ with $i \in\{1, \ldots, 13\}$ indicates a subset of $\mathbf{F}$ that has $\left(\begin{array}{c}13 \\ i\end{array}\right)$ feature sets and each feature set contains a combination of $i$ metrics. In the following paragraphs, we explain the classification approach for an exemplary feature set, $\mathrm{f}_{\mathrm{e}}$, selected from F. The train and test data sets required for classification were constructed by choosing $\mathrm{f}_{\mathrm{e}}$ of all participants from $\mathrm{S}_{\text {norm }}$ and $\mathrm{M}_{\text {norm }}$ and we called them $\mathrm{S}_{\text {train }}$ and $\mathrm{M}_{\text {test }}$, respectively. The proposed forward-backward classification method, comprised of two identical k-nearest-neighbor (kNN: $k=1$, Euclidean distance) classifiers. classifier (1) in forward classification was trained by $\mathrm{S}_{\text {train }}$ in six classes with known class labels from the model simulations $\left(\mathrm{L}_{\mathrm{S}}\right)$, that is, the six simulated CS profiles previously described in Figure 3. Then, individual CS profiles were predicted by testing the trained classifier with the $\mathrm{M}_{\text {test }}$. Figure 6A visualizes the different steps in forward classification. In this step, the evaluation of classification performance is unfeasible, since the actual CS degree of experiment participants are unknown. To address this issue, we interchanged the train-test data sets of the forward classification and implemented a second classification approach, called backward classification to assess the performance of the classifier (1) based on a second classifier (Figure 
6B). In this regard, we took the output of forward classification, that is, the predicted CS degrees of experiment participants ( $\mathrm{L}_{\mathrm{M}}$ in Figure 6), and corresponding measured AEP metrics $\left(\mathrm{M}_{\text {test }}\right)$ to train the classifier (2) of Figure 6B. Afterwards, $\mathrm{S}_{\text {train }}$, with known CS labels $\left(\mathrm{L}_{\mathrm{S}}\right)$ from the simulated individualized CS profiles, was used to test the trained classifier (2). The vector of predicted CS labels by classifier (2) $\left(\mathrm{L}_{\mathrm{S}_{\text {Pred }}}\right)$ was compared to $\mathrm{L}_{\mathrm{S}}$ and correspondig prediction accuracy was calculated as follows

$$
\text { acc }=\frac{\sum_{q=1}^{n}\left[L_{S}(q)==L_{S_{\text {Pred }}}(q)\right]}{n}
$$

where $n$ is equal to 210 ( 35 subjects with six CS profiles). Thus, the backward classification offers the possibility to calculate the accuracy of predicted CS profiles of study participants based on model simulations. We then repeated the forward-backward classification over all possible combinations of the derived metrics, that is, 8,191 feature-sets and calculated the prediction accuracy of each feature set according to Equation 16. In this respect, the backward classification method gives the insight that to which degree classifier (1) was accurate in predicting CS degrees of experimental participants. Our classification approach makes use of combined simulated and recorded data to predict CS profiles and can test the accuracy of these methods, even though a direct and actual validation of the CS histopathology still remains hidden due to experimental difficulties.

\section{Results}

We applied forward-backward classification for each of the cochlear model individualization methods (AudTH and DPTH) and calculated the prediction accuracy of all feature-sets in $\mathbf{F}$. For each cochlear profiling method, first, we determined the feature-set in each $\mathrm{F}_{\mathrm{i}}(i \in\{1, \ldots, 13\})$ that had the highest classification accuracy. $F_{i}$ consisted of feature sets with $i$ AEP-derived metrics. Then, the prediction variability was estimated using forward-backward classification by including the standard deviations of selected feature-sets. Finally, we report individually predicted CS profiles belonging to those feature sets.

\section{Combination of AEP-Derived Metrics}

To determine the best combination of metrics for CS profiling, the forward-backward classification was performed on the mean AEP-derived metrics of experiment participants and corresponding classification accuracy was reported as accmean. Thus, we calculated acc mean values of the predictions for 8,191 feature sets in $\mathbf{F}$ and determined the feature set that yielded the highest acc $_{\text {mean }}$ among all feature-sets in $F_{i}$, with $\mathrm{i}$ combined metrics $(i \in\{1, \ldots, 13\})$. Accordingly, 13 feature sets were selected among 8,191 in F. Tables 2 and 3 list those feature sets and corresponding acc $\mathrm{c}_{\text {mean }}$ values for

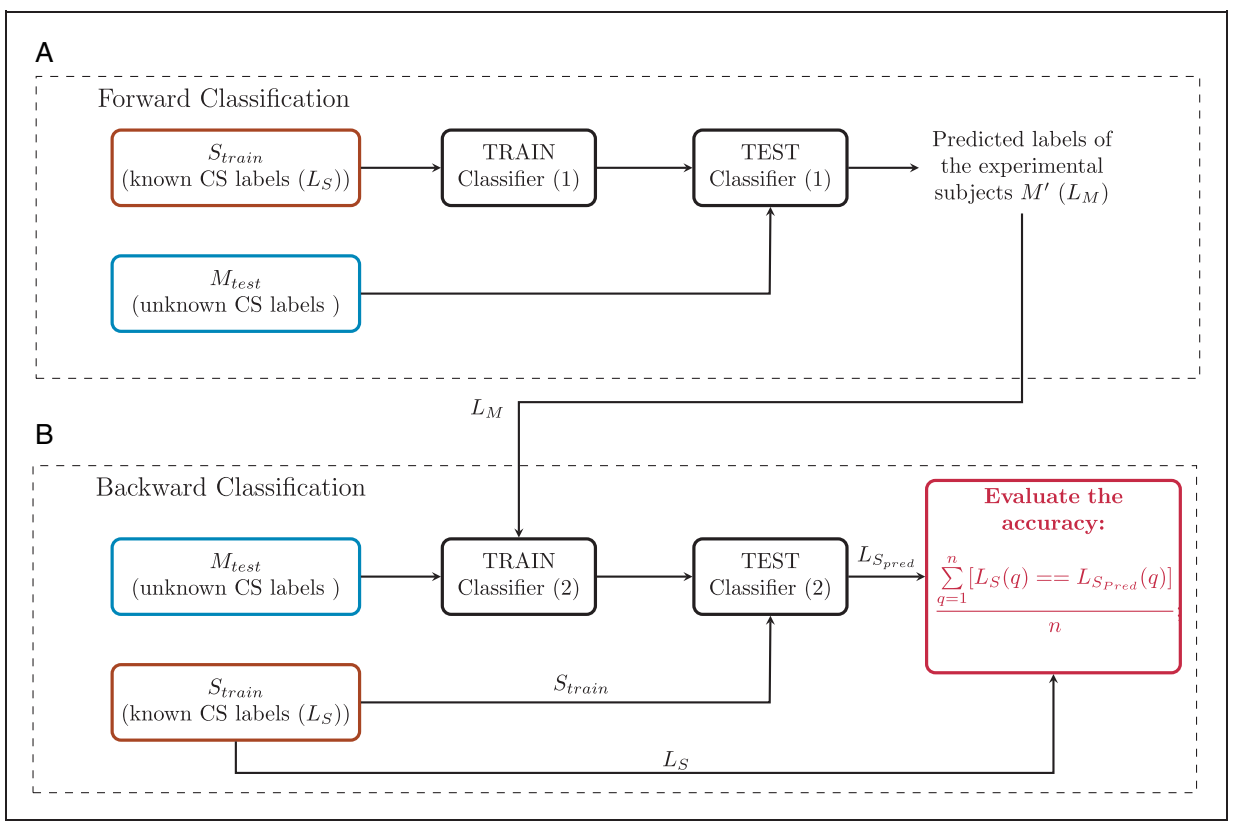

Figure 6. The Forward-Backward Classification Method. A: Forward classification: Classifier (I) is trained with individualized simulated AEP-derived metrics $\left(S_{\text {train }}\right)$ for six CS profiles $\left(L_{S}\right)$ and tested with measured AEP-derived metrics $\left(M_{\text {test }}\right)$. The predicted labels $\left(L_{M}\right)$ for the study participants are entered to block (B). The backward-classification in (B) trains classifier (2) using measured AEP-derived metrics, that is, $M_{\text {(test) }}$, and labels predicted by the forward classification, that is, $L_{M}$. Classifier (2) is tested by $S_{\text {train }}$ and corresponding labels ( $\left.L_{S}\right)$ are used to assess the classifier performance. $\mathrm{CS}=$ cochlear synaptopathy. 
AudTH and DPTH-based methods, respectively. The RAM-EFR metric yielded the highest $\mathrm{acc}_{\text {mean }}$ values for both cochlear model individualization methods. The obtained $83.81 \%$ acc $_{\text {mean }}$ of DPTH-based method was higher than that of the AudTH-based method (68.57\%), suggesting that methods which assess OHC damage more directly (i.e., DPTH vs. AudTH) yield a better classification accuracy in predicting simulated individualized CS profiles.

\section{Prediction Variability}

The impact of subject-specific factors and measurement noise reflect on inter- and within-subject variability of the AEP recordings and can have an impact on the accuracy of the classification method. To measure this effect, the forward-backward classification was repeated, this time by extracting metrics from the bootstrapped average trials, rather than from the mean of trials. This resulted in distributions for each specific metric and each subject, with standard deviations as given by the last column of Table 1 . Then, 100 samples were randomly drawn from the distribution of each metric. Thus, for every feature-set in Tables 2 and 3, the corresponding metrics samples were combined to yield 100 variations of each feature-set. Afterwards, the CS profile prediction was repeated 100 times with each feature-set for each subject, and prediction accuracy was assessed in every repetition. Lastly, the standard deviation of the calculated accuracies $\left(\mathrm{acc}_{\mathrm{SD}}\right)$ was determined over the 100 repetitions of each feature-set and listed in the last column of Tables 2 and 3 .

For the best predictor metric (RAM-EFR), acc $\mathrm{CD}_{\mathrm{SD}}$ values of $2.95 \%$ and $2.66 \%$ were obtained for the AudTH- and DPTH-based methods, respectively. The lowest acc $_{\mathrm{SD}}$ was obtained when combining the RAMEFR with the $\mathrm{w}-\mathrm{V}_{\text {lat100 }}$ metric in both cochlear model individualization methods (AudTH: $1.73 \%$ and DPTH: $1.34 \%)$. However, the respective $\mathrm{acc}_{\text {mean }}$ values were considerably lower than those of the RAM-EFR by itself, particularly in DPTH-based method. To assess the performance of the RAM-EFR based CS profile prediction in subgroups, we show confusion tables in Figure 7 for AudTH- and DPTH-based cochlear model individualization methods. The diagonals of each table reflect how often the classifier assigned a CS profile $\left(\mathrm{L}_{\mathrm{S}_{\text {Pred }}}\right.$ : predicted class) that matched with that of in simulated individualized CS profiles ( $\mathrm{L}_{\mathrm{S}}$ : true class). Off-diagonal values show the number of instances that $\mathrm{L}_{\mathrm{S}_{\text {Pred }}}$ and $\mathrm{L}_{\mathrm{S}_{\text {Pred }}}$ were not identical. Detailed prediction accuracy values of each subgroup are summarized in the

Table 2. Combination of Metrics With the Highest Mean Accuracy $\left(\mathrm{acc}_{\text {mean }}\right)$ Values in Each $\mathrm{F}_{\mathrm{i}}$, With $i$ Combined Metrics. The reported results are based on AudTH-based cochlear model individualization method.

\begin{tabular}{|c|c|c|c|c|}
\hline \multirow{2}{*}{$\begin{array}{l}\text { Involved } \\
\text { metrics }\end{array}$} & \multirow{2}{*}{$\begin{array}{l}\text { Involved } \\
\text { subjects }\end{array}$} & \multirow[b]{2}{*}{ Best combination of metrics } & \multicolumn{2}{|c|}{$\operatorname{acc}(\%)$} \\
\hline & & & $\mathrm{acc}_{\text {mean }}$ & $\operatorname{acc}_{s d}$ \\
\hline I & 35 & RAM-EFR & 68.57 & 2.95 \\
\hline 2 & 35 & RAM-EFR, $w-V_{\text {lat } 100}$ & 64.76 & 1.73 \\
\hline 3 & 35 & RAM-EFR, w-I lat $100, w-I_{100}$ & 53.33 & 7.86 \\
\hline 4 & 35 & RAM-EFR, w- $\mathrm{V}_{\text {lat } 100}, \mathrm{w}-\mathrm{I}_{\text {lat } 100}, \mathrm{w}-\mathrm{I}_{100}$ & 51.90 & 9.28 \\
\hline 5 & 35 & RAM-EFR, w- $V_{\text {lat } 100}, w-I_{\text {lat } 100}, w-I_{100}, w-V_{70}$ & 52.86 & 8.69 \\
\hline 6 & 35 & RAM-EFR, w- $\left.\right|_{\text {lat } 100}, w-I_{100}, w-V_{70}, w-I_{70}, w-V$-growth & $5 \mathrm{I} .43$ & 6.97 \\
\hline 7 & 35 & $\begin{array}{l}\text { RAM-EFR, w- } V_{\text {lat }}-\text { growth, w-V-growth, } \\
w-V_{\text {lat } 100}, w-I_{70}, w-V_{70}, w-V_{100}\end{array}$ & 45.24 & 6.79 \\
\hline 8 & 35 & $\begin{array}{l}\text { RAM-EFR, w-V } V_{\text {lat }}-\text { growth, w-V-growth, } \\
w-V_{\text {lat } 100, w-V_{\text {lat } 70}, w-I_{70}, w-V_{70}, w-V_{100}}\end{array}$ & 45.24 & 6.59 \\
\hline 9 & 35 & $\begin{array}{l}\text { RAM-EFR, w-V-growth, w-I-growth, } \\
\quad w-I_{\text {lat } 100}, w-V_{\text {lat } 100}, w-I_{70}, w-V_{70}, w-I_{100}, w-V_{100}\end{array}$ & 36.19 & 7.11 \\
\hline 10 & 35 & $\begin{array}{l}\text { RAM-EFR, w-V-growth, w-l-growth, w- } V_{\text {lat }}-\text { growth, } \\
w-V_{\text {lat } 100}, w-V_{\text {lat } 70}, w-I_{70}, w-V_{70}, w-I_{100}, w-V_{100}\end{array}$ & 32.86 & 6.67 \\
\hline 11 & 35 & $\begin{array}{l}\text { RAM-EFR, w-V-growth, w-l-growth, w- } \mathrm{V}_{\text {lat }}-\text { growth, w-I lat }- \text { growth, } \\
\mathrm{w}-\mathrm{V}_{\text {lat } 100}, \mathrm{w}-\mathrm{V}_{\text {lat } 70}, \mathrm{w}-\mathrm{I}_{70}, \mathrm{w}-\mathrm{V}_{70}, \mathrm{w}-\mathrm{I}_{100}, \mathrm{w}-\mathrm{V}_{100}\end{array}$ & 27.62 & 6.49 \\
\hline 12 & 35 & $\begin{array}{l}\text { RAM-EFR, w-V-growth, w-l-growth, w- } V_{\text {lat }}-\text { growth, w-I lat }- \text {-growth, } \\
w-V_{\text {lat } 100}, w-V_{\text {lat } 70}, w-I_{70}, w-V_{70}, w-I_{100}, w-V_{100}, w-I_{\text {lat70 }}\end{array}$ & 18.10 & 6.65 \\
\hline 13 & 35 & $\begin{array}{l}\text { RAM-EFR, w-V-growth, w-I-growth, w- } \mathrm{V}_{\text {lat }}-\text { growth, w- } \mathrm{I}_{\text {lat }}-\text { growth, } \\
\mathrm{w}-\mathrm{V}_{\text {lat } 100}, \mathrm{w}-\mathrm{V}_{\text {lat70 }}, \mathrm{w}-\mathrm{I}_{70}, \mathrm{w}-\mathrm{V}_{70}, \mathrm{w}-\mathrm{I}_{100}, \mathrm{w}-\mathrm{V}_{100}, \mathrm{w}-\mathrm{I}_{\text {lat } 70}, \mathrm{w}-\mathrm{I}_{\text {lat } 100}\end{array}$ & 17.14 & 6.75 \\
\hline
\end{tabular}

Note. $\mathrm{EFR}=$ envelope-following response. The standard deviations of obtained accuracies are shown in acc sd $_{\text {column. }}$ 
Table 3. Combination of Metrics With the Highest Mean Accuracy ( acc $_{\text {mean }}$ ) Values in Each $\mathrm{F}_{\mathrm{i}}$, With $i$ Combined Metrics. The reported results are based on DPTH-based cochlear model individualization method.

\begin{tabular}{|c|c|c|c|c|}
\hline \multirow{2}{*}{$\begin{array}{l}\text { Involved } \\
\text { metrics }\end{array}$} & \multirow{2}{*}{$\begin{array}{l}\text { Involved } \\
\text { subjects }\end{array}$} & \multirow{2}{*}{ Best combination of metrics } & \multicolumn{2}{|l|}{ acc $(\%)$} \\
\hline & & & $\mathrm{acc}$ mean & $\mathrm{acc}_{\mathrm{sd}}$ \\
\hline I & 35 & RAM-EFR & 83.81 & 2.66 \\
\hline 2 & 35 & RAM-EFR, w- $V_{\text {lat } 100}$ & 58.57 & 1.34 \\
\hline 3 & 35 & RAM-EFR, w- $\left.\right|_{\text {lat } 100,}, w-I_{100}$ & 54.29 & 8.34 \\
\hline 4 & 35 & RAM-EFR, w- $\mathrm{V}_{\text {lat } 100}, \mathrm{w}-\mathrm{I}_{\text {lat } 100}, \mathrm{w}-\mathrm{I}_{100}$ & 61.90 & 8.22 \\
\hline 5 & 35 & RAM-EFR, w- $\mathrm{V}_{\text {lat } 100}, \mathrm{w}-\mathrm{I}_{\text {lat } 100}, \mathrm{w}-\mathrm{I}_{100}, \mathrm{w}-\mathrm{V}_{70}$ & 58.10 & 8.90 \\
\hline 6 & 35 & RAM-EFR, w- $\mathrm{V}_{\text {lat } 100}, \mathrm{w}-\mathrm{I}_{\text {lat } 100}, \mathrm{w}-\mathrm{I}_{100}, \mathrm{w}-\mathrm{V}_{70}, \mathrm{w}$-I-growth & 48.10 & 7.40 \\
\hline 7 & 35 & RAM-EFR, w- $\mathrm{V}_{\text {lat } 100}, w-\mathrm{l}_{\text {lat } 100}, w-\mathrm{V}_{100}, w-\mathrm{V}_{70}, \mathrm{w}-\mathrm{V}$-growth, $w-\mathrm{I}_{70}$ & 40.95 & 6.96 \\
\hline 8 & 35 & RAM-EFR, w- $V_{\text {lat } 100}, w-I_{\text {lat } 100}, w-V_{100}, w-V_{70}, w-I-g r o w t h, ~ w-I_{70}, w-V_{100}$ & 35.71 & 7.12 \\
\hline 9 & 35 & $\begin{array}{l}\text { RAM-EFR, w-I-growth, w-V-growth, w- } V_{\text {lat }} \text {-growth, w-I lat70 }, w-I_{70} \text {, } \\
w-V_{70}, w-I_{100}, w-V_{100}\end{array}$ & 34.29 & 7.30 \\
\hline 10 & 35 & $\begin{array}{l}\text { RAM-EFR, w-I-growth, w-V-growth, w- } \mathrm{V}_{\text {lat }} \text {-growth, w-I lat70, w- } \mathrm{V}_{\text {lat70 }}, \\
w-\mathrm{V}_{\text {lat } 100}, w-\mathrm{I}_{70}, w-\mathrm{I}_{100}, w-\mathrm{V}_{100}\end{array}$ & 29.52 & 6.53 \\
\hline II & 35 & $\begin{array}{l}\text { RAM-EFR, w-I-growth, w-V-growth, w- } V_{\text {lat }}-\text { growth, w- }-V_{\text {lat70 }} \\
\quad w-I_{\text {lat } 100}, w-V_{\text {lat } 100}, w-I_{70}, w-I_{100}, w-V_{70}, w-V_{100}\end{array}$ & 17.14 & 6.13 \\
\hline 12 & 35 & $\begin{array}{l}\text { RAM-EFR, w-I-growth, w-V-growth, w- } \mathrm{V}_{\text {lat }}-\text { growth, w-I lat }- \text { growth, w-I lat70, } \\
w-V_{\text {lat70 }}, w-V_{\text {lat } 100}, w-I_{\text {lat } 100}, w-I_{70}, w-V_{70}, w-I_{100}\end{array}$ & 16.67 & 2.63 \\
\hline 13 & 35 & $\begin{array}{l}\text { RAM-EFR, w-V-growth, w-I-growth, w- } \mathrm{V}_{\text {lat }}-\text { growth, w-l lat }- \text { growth, } \\
\mathrm{w}-\mathrm{V}_{\text {lat } 100}, \mathrm{w}-\mathrm{V}_{\text {lat } 70}, \mathrm{w}-\mathrm{I}_{70}, \mathrm{w}-\mathrm{V}_{70}, \mathrm{w}-\mathrm{I}_{100}, \mathrm{w}-\mathrm{V}_{100}, \mathrm{w}-\mathrm{I}_{\text {lat } 70}, \mathrm{w}-\mathrm{I}_{\text {lat } 100}\end{array}$ & 16.67 & 2.93 \\
\hline
\end{tabular}

Note. EFR = envelope-following response. The standard deviations of obtained accuracies are shown in acc $c_{s d}$ column.

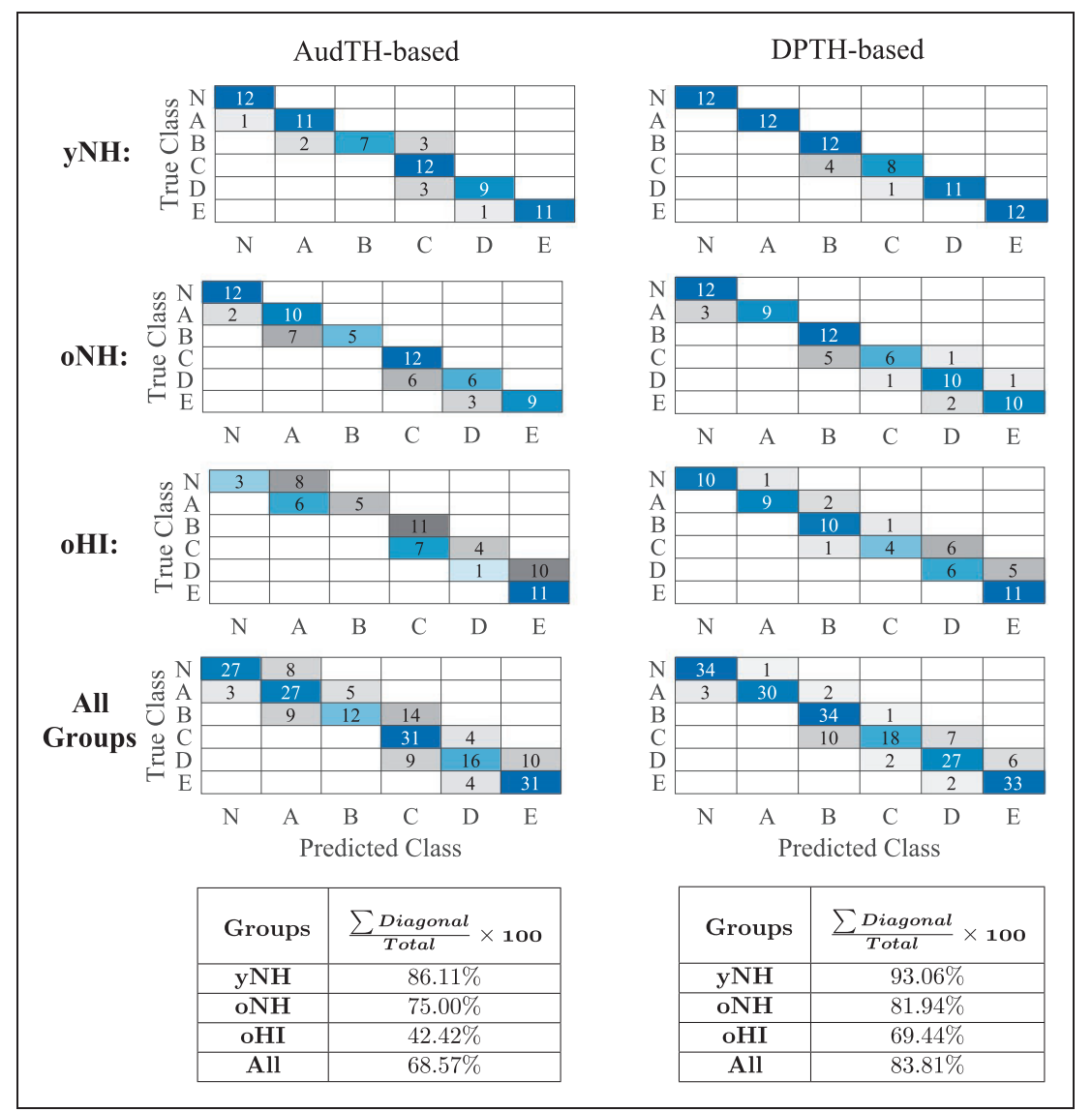

Figure 7. Confusion Tables at Subgroup and Group-Levels for Both AudTH and DPTH-Based Cochlear Model Individualization Methods. The tables summarize the accuracy of classifier (2) in Figure 6B for subgroups as well as all groups together. AudTH $=$ audiometric thresholds; DPTH = DPOAE threshold. 
tables in Figure 7. The highest and lowest prediction accuracy values relate to the $\mathrm{yNH}$ and oHI group, respectively for both AudTH- and DPTH-based methods. Comparing the cochlear model individualization methods, it is seen that the DPTH-based approach outperforms the AudTH-based method on both group- and subgroup levels.

\section{CS Profile Prediction Based on Individualized Classifiers}

Table 4 lists the predicted individual CS profiles from the RAM-EFR metric (best prediction accuracy) for both AudTH- and DPTH-based cochlear individualization methods. The reported profiles are the output of the forward classification step, that is, $\mathrm{L}_{\mathrm{M}}$ shown in Figure 6. Considering the AudTH and DPTH columns of Table 4, lower degrees of AN damage were predicted for the yNH group than for the oNH and oHI groups. In addition, the range of predicted CS profiles in the $\mathrm{yNH}$ group shows that yNH listeners might also suffer from different degrees of CS. The oHI group, which was assumed to suffer from mixed $\mathrm{OHC}$ damage and CS pathologies, were predicted to have the highest degree of CS among the cohort.

Thus far, the reported individualized CS profiles for RAM-EFR were predicted by training a single classifier with simulated individualized CS profiles of the whole experimental cohort. This has drawbacks for individual profiling in a clinical context, because it would be ideal if the profiling could be performed using only recordings from the tested individual. Hence, to establish more accurate predictions of the individual CS degrees, we took one step further and designed individualized classifiers, which were trained and tested with the RAMEFR metric of the same listener. If $\mathrm{RAM}_{\mathrm{s}}$ stands for the six simulated CS profiles of a nominal subject and $\mathrm{RAM}_{\mathrm{m}}$ for the measured RAM-EFR metric, we first normalized $\mathrm{RAM}_{\mathrm{s}}$ and $\mathrm{RAM}_{\mathrm{m}}$ values by the $\overline{\mathrm{RAM}_{\mathrm{s}}}$ and $\sigma_{\mathrm{RAM}_{\mathrm{s}}}$ (mean and standard deviation of $\mathrm{RAM}_{\mathrm{s}}$ ). Then we trained and tested the classifier, with the same characteristics as classifiers (1) and (2), using normalized $\mathrm{RAM}_{\mathrm{s}}$ and $\mathrm{RAM}_{\mathrm{m}}$ values, respectively. This procedure was repeated for all listeners in the cohort and for both AudTH and DPTH-based cochlear model pole-setting methods. The predicted individualized CS profiles were listed in Table 4 (columns: $\mathrm{AudTH}_{\text {ind }}$ and $\left.\mathrm{DPTH}_{\text {ind }}\right)$. Considering either of the AudTH- or DPTHbased methods, designing individualized classifiers revealed only minor differences in the predicted CS profiles of individual listeners compared to those predicted by a single classifier trained with simulated individualized RAM-EFRs. However, the CS profiles reported in AudTH $\mathrm{Hind}_{\text {and }} \mathrm{DPTH}_{\text {ind }}$ columns might be more reliable than the group-based predictions, since the former were predicted by individualized classifiers that were trained on the basis of personalized cochlear simulations.

To provide a demonstration of the implemented method, and to show to which extent the model simulations imitate the experimental measurements, we compare simulated and measured AEPs of a yNH subject in Figure 8. Panel A depicts simulated RAM-EFR spectra for the different considered CS profiles. Based on the experimental RAM-EFR (Panel D) and forward classification, we predicted that this subject had a "N" CS profile, that is, no AN damage. The CGL parameters of the individualized model were adjusted based on DPTHs of the same yNH listener. Panels $\mathrm{B}$ and $\mathrm{C}$

Table 4. Predicted Individuals CS Profiles Obtained From AudTH and DPTH-Based Cochlear Individualization Methods, Based on RAM-EFR Metric.

\begin{tabular}{|c|c|c|c|c|c|}
\hline Group & No. & AudTH & AudTH $_{\text {ind }}$ & DPTH & DPTH $_{\text {ind }}$ \\
\hline & I & C & $B$ & B & B \\
\hline & 2 & $A$ & $A$ & $A$ & B \\
\hline & 5 & $\mathrm{~N}$ & $\mathrm{~N}$ & $\mathrm{~N}$ & $\mathrm{~N}$ \\
\hline & 7 & $N$ & $\mathrm{~N}$ & $\mathrm{~N}$ & $N$ \\
\hline \multirow[t]{12}{*}{$y \mathrm{NH}$} & 8 & $\mathrm{~N}$ & $\mathrm{~N}$ & $\mathrm{~N}$ & $\mathrm{~N}$ \\
\hline & 9 & $\mathrm{~N}$ & $\mathrm{~N}$ & $\mathrm{~N}$ & $\mathrm{~N}$ \\
\hline & 10 & $\mathrm{~N}$ & $\mathrm{~N}$ & $N$ & $A$ \\
\hline & II & $A$ & B & $B$ & B \\
\hline & 12 & $\mathrm{~N}$ & $\mathrm{~N}$ & $\mathrm{~N}$ & A \\
\hline & 13 & $A$ & $A$ & A & A \\
\hline & 14 & $\mathrm{~N}$ & $\mathrm{~N}$ & $\mathrm{~N}$ & $N$ \\
\hline & 15 & $\mathrm{~N}$ & $\mathrm{~N}$ & $\mathrm{~N}$ & $N$ \\
\hline & 1 & $D$ & $D$ & $C$ & $D$ \\
\hline & 3 & $E$ & $E$ & $E$ & $E$ \\
\hline & 4 & $D$ & $E$ & $D$ & $D$ \\
\hline & 6 & $D$ & $D$ & $D$ & $D$ \\
\hline \multirow[t]{13}{*}{ oNH } & 7 & C & $D$ & $D$ & $\mathrm{D}$ \\
\hline & 8 & $E$ & $E$ & $E$ & $E$ \\
\hline & 9 & $\mathrm{~N}$ & $A$ & $\mathrm{~N}$ & $A$ \\
\hline & 10 & B & $B$ & $B$ & $B$ \\
\hline & II & $\mathrm{C}$ & $D$ & $\mathrm{D}$ & $\mathrm{D}$ \\
\hline & 12 & $\mathrm{~N}$ & $\mathrm{~N}$ & $\mathrm{~N}$ & $\mathrm{~N}$ \\
\hline & 13 & $E$ & $E$ & $E$ & $E$ \\
\hline & 14 & C & $D$ & C & C \\
\hline & I & $E$ & $E$ & $E$ & $E$ \\
\hline & 2 & $E$ & $D$ & $E$ & $\mathrm{D}$ \\
\hline & 3 & $E$ & $E$ & $E$ & $\mathrm{E}$ \\
\hline & 4 & $E$ & $E$ & $E$ & $E$ \\
\hline & 5 & $E$ & $\mathrm{D}$ & $E$ & $E$ \\
\hline \multirow[t]{6}{*}{$\mathrm{oHI}$} & 7 & $E$ & $D$ & $E$ & $E$ \\
\hline & 8 & $E$ & $E$ & $E$ & $E$ \\
\hline & 9 & $\mathrm{E}$ & $\mathrm{E}$ & $E$ & $E$ \\
\hline & 10 & $\mathrm{E}$ & $\mathrm{E}$ & $E$ & $E$ \\
\hline & 11 & $\mathrm{E}$ & $E$ & $E$ & $E$ \\
\hline & 12 & $\mathrm{E}$ & $\mathrm{E}$ & $E$ & $E$ \\
\hline
\end{tabular}

Columns AudTH $_{\text {ind }}$ and DPTH $\mathrm{Hind}_{\text {ist }}$ lise predicted $\mathrm{CS}$ profiles by designing individualized classifiers based on RAM-EFR metric. 


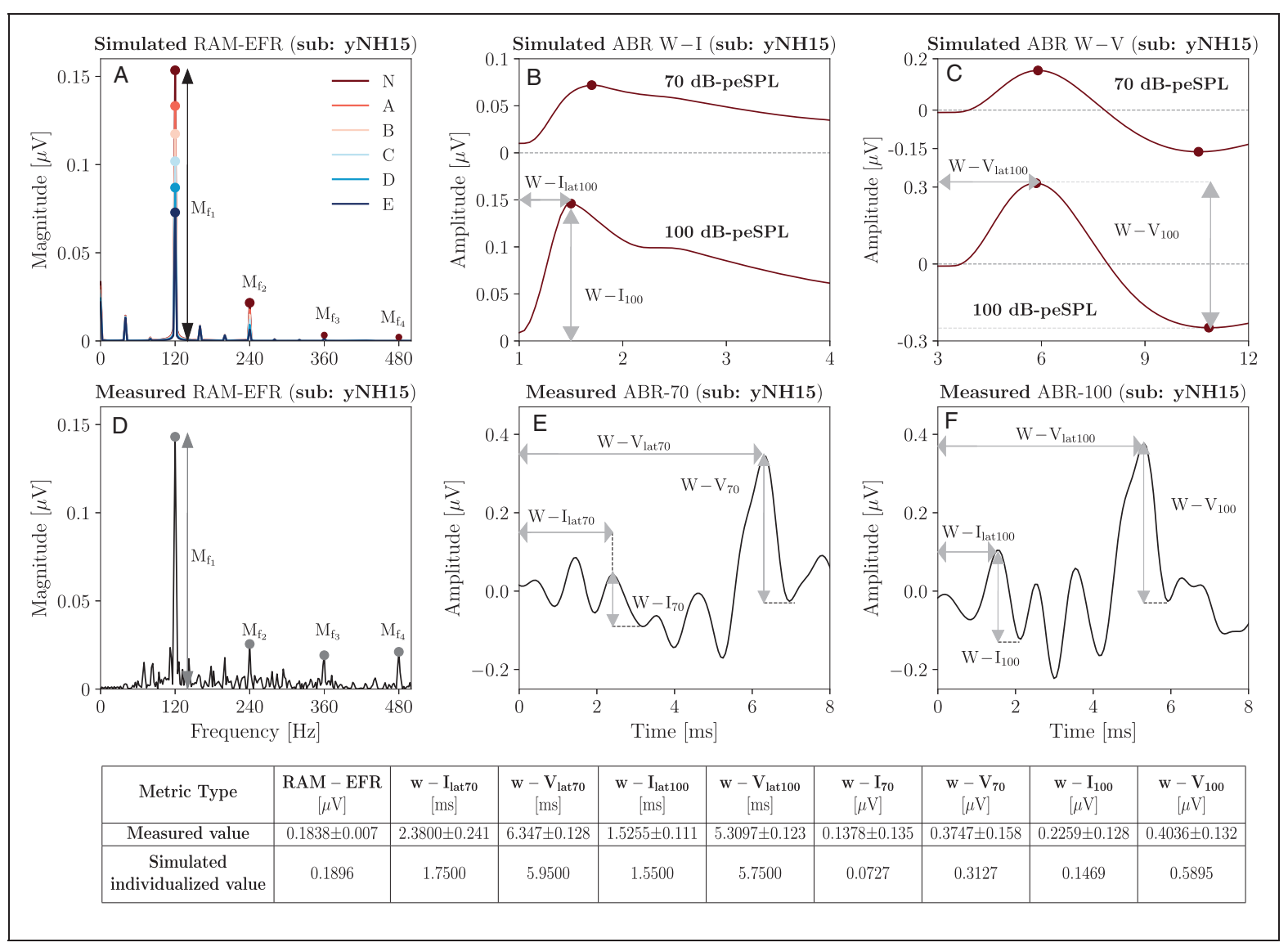

Figure 8. A Comparison Between Simulated and Measured AEPs for a yNH Subject (yNHI5). This subject was predicted to have a normal (N) CS profile, that is, without CS. A: Simulated RAM-EFR spectra for six CS profiles. The sum of the drawn arrows yields the RAM-EFR magnitude metric. B: Simulated ABR wave-I to 70 and $100 \mathrm{~dB}$-peSPL clicks. Waveforms were shifted by I ms to match the experimental data. C: Simulated ABR wave- $V$ to 70 and $100 \mathrm{~dB}$-peSPL clicks. Waveforms were shifted by 3 ms to match the experimental data. The specified arrows in (B) and (C) indicate the extracted metrics. D: Measured RAM-EFR of the same listener (yNHI5). Shown arrows, indicate the peak-to-noisefloor values. Akin to (A), the measured RAM-EFR metric was calculated by summing the arrow amplitudes. E: Measured ABR waveform to $70 \mathrm{~dB}$-peSPL clicks. F: Measured ABR waveform to $100 \mathrm{~dB}$-peSPL clicks. Arrows in (E) and (F) determine the extracted metrics. The shown simulated waveforms were predicted based on the DPTH-based cochlear individualization method. The table shows the exact value of EFR and ABR metrics derived from recordings and predicted CS-profile, "N," of the same listener. $E F R=$ envelope-following response; $A B R=$ auditory brainstem response; $C S=$ cochlear synaptopathy.

depict the simulated personalized ABR waveforms for the predicted "N" CS degree. Experimental ABR waveforms to 70 and $100 \mathrm{~dB}$-peSPL clicks are shown in Panels (E) and (F), respectively. Details regarding the value of extracted metrics from the measurements and simulations are provided in a table at the bottom of Figure 8. Even though our classifier did not consider ABR metrics, the applied personalized $\mathrm{OHC}$ and $\mathrm{AN}$ profiles predicted $\mathrm{w}-\mathrm{I}_{\text {lat } 100}, \mathrm{w}-\mathrm{I}_{70}, \mathrm{w}-\mathrm{V}_{70}$ and $\mathrm{w}-\mathrm{I}_{100}$ markers that fell the standard deviation of the corresponding recorded values. The remaining simulated ABR metrics, that is, $\mathrm{w}-\mathrm{I}_{\text {lat70 }}, \mathrm{w}-\mathrm{V}_{\text {lat100, and }}$ $\mathrm{w}-\mathrm{V}_{100}$, only minimally deviated from the range of respective measurements, showing that our method accurately predicts AEP features to stimuli which were not included in the classifier.

\section{Method Validation}

To validate the proposed method and its generalizability to other cohorts and other measurement equipment, we applied the developed classifier in backward classification step to RAM-EFRs recorded in a second experiment. Figure 9 schematizes the implementation of the validation method. Considering the different experimental setup and recording location of the second experiment, the measured RAM-EFRs of both experiments were scaled between zero and one, prior to classification. 


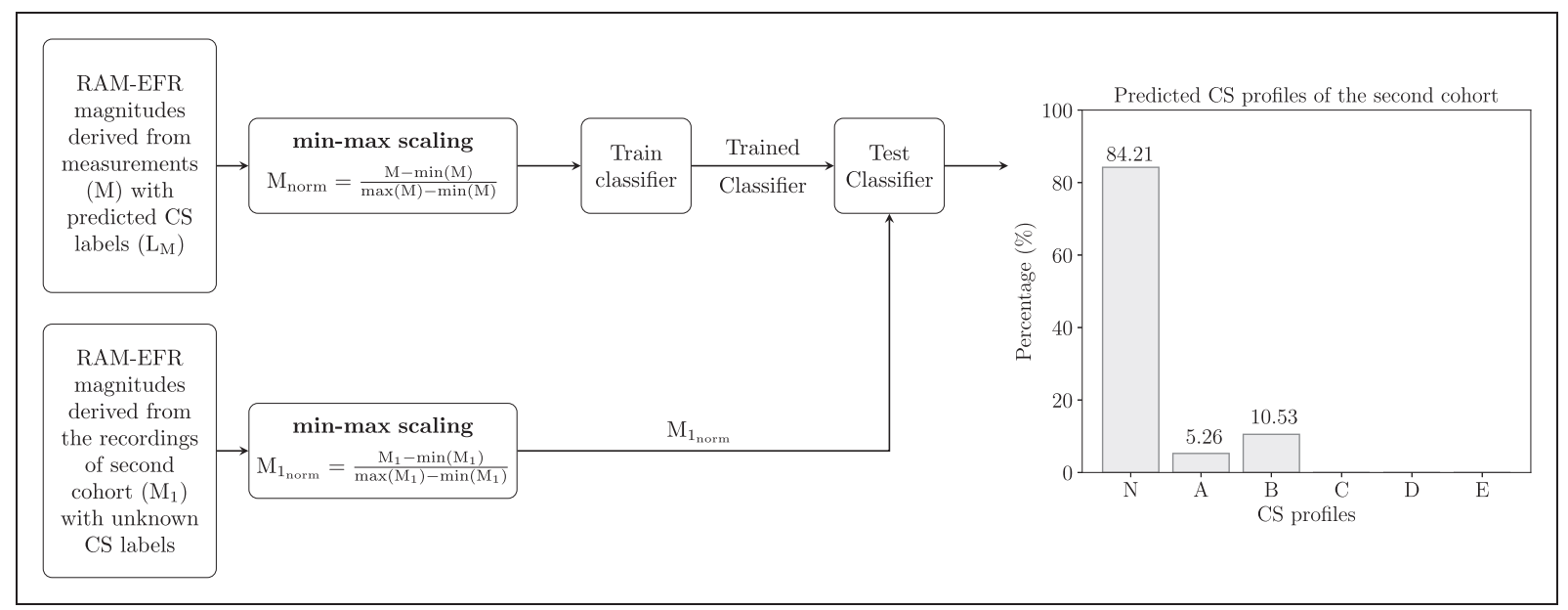

Figure 9. Implementation of the Validation Method. Measured RAM-EFRs (M) with predicted labels in Figure 6 ( $\left.L_{M}\right)$ are scaled between zero and one to train a kNN classifier. The trained classifier is tested with scaled RAM-EFRs recorded from the second cohort comprised of $\mathrm{yNH}$ listeners. The bar-plot shows the predicted CS profiles for the second cohort listeners. The CS profiles labels in the bar-plot are similar to those defined in Figure 3. EFR = envelope-following response; CS=cochlear synaptopathy.

Given that only yNH listeners participated in the second experiment, we employed the smallest RAM-EFR magnitude recorded from oHI listeners (as part of another study) recorded with the same setup as the second experiment to scale the RAM-EFRs. The scaled RAM-EFRs of the first experiment were used to train the classifier (1) in Figure 6 and afterwards, the trained classifier was tested with the scaled RAM-EFRs of the second experiment. The predicted CS profiles are illustrated as a barplot in Figure 9. Moreover, 84.21\% of the 19 yNH participants of the second experiment were classified as $N$, that is, without CS, and the rest were predicted to have mild CS. These predictions show that a classifier designed on our cohort can be applied to other cohorts to predict individual CS degrees based on the RAMEFR. In line with expectations, the classifier predicted that most yNH subjects were synaptopathy free.

\section{Discussion}

By combining experimental ABR and EFR measurements with a modeling approach, we were able to develop a classifier that can assign one out of six CS profiles to listeners with mixed SNHL pathologies. The classifier considered 8,191 feature sets, of which our forwardbackward classification method identified that the RAM-EFR metric yielded the best performance in both AudTH- and DPTH-based cochlear individualization methods. We tested both a group and individually based method and showed that our method can generalize to other cohorts and measurement setups. Taken together, we have high hopes that this method can find its way to clinical hearing diagnostics, since a single AEP metric is required to yield a CS-profile prediction, given the audiogram or at least four DPTHs.

\section{Implications for RAM-EFR-Based Synaptopathy Profiling Prediction}

On the one hand, predicting the CS degree from AEP metrics is controversial in listeners with coexisting $\mathrm{OHC}$ deficits and on the other, validation of the predicted CS profiles with temporal bone histology is impossible in humans. Without these means, models of the human auditory periphery and AEP generators can provide a tool to bridge this experimental gap. The similarity between predicted AEP degradations for a known CS profile and experimental AEP degradations can be used to predict the CS profile of individuals. In a previous study, we tested the potential of the derived-band EFR as a CS predictor in NH listeners using a fuzzy c-means clustering method and validated our CS predictions using an another AEP-derived metric (wave-V amplitude growth slope) recorded from the same listener. We evaluated the method based on the percent of subjects that were predicted and validated to have the same CS profile, that is, $61 \%$ (Keshishzadeh \& Verhulst, 2019). However, the performance of this method is easily impacted by the characteristics of the adopted predictor and validation metrics, for example, different generator sources, degree of sensitivity to subtypes of SNHL, and tonotopic susceptibility.

The interdisciplinary approach we took in this study tackled this validation issue by proposing a forwardbackward classification approach and applying the trained classifier to AEPs from a new cohort to test its generalizability. Moreover, we were able to determine the most accurate AEP-derived metric for CS degree 
prediction, given a range of 13 possible AEP-derived metrics. Among the considered AEP-based metrics and combinations thereof, we found that the RAM-EFR magnitude showed the best performance in segregating simulated individual CS profiles. At the same time, RAM-EFR metric was involved in all feature sets that yielded the highest $\mathrm{acc}_{\text {mean }}$ among feature sets that had equal number of combined metrics (Tables 2 and 3 ). This finding is consistent with the outcome of Parthasarathy and Kujawa (2018) and Vasilkov et al. (2021), showing that EFRs to SAM or RAM are sensitive to CS. Moreover, the combined modeling and experimental study of Vasilkov et al. (2021) showed that the adopted RAM-EFR marker (RAM with a $25 \%$ duty cycle) is minimally impacted by OHC damage. The sharp envelope combined with the long silence intervals between stimulus peaks generates more synchronized AN fiber responses compared to conventional SAM stimulus to yield a stronger EFR with extended dynamic range across subjects. Finally, the RAM-EFR is a more sensitive marker of CS than ABR (Parthasarathy \& Kujawa, 2018). Taken together, our results indicate that the RAM-EFR magnitude is an appropriate AEP-based metric to predict individual CS degree of listeners in the presence of OHC-loss.

\section{The Effect of Cochlear Model Individualization Method on Predicting Cochlear Synaptopathy Profiles}

In this study, we determined the CGL model parameters using either measured audiometric or DPOAE thresholds and assessed the classifier performance of each method in the backward classification step. Comparing the resulting $\mathrm{acc}_{\text {mean }}$ values for each cochlear individualization method can infer which of the two methods yielded the most accurate AEP simulations for a given CS profile. The accmean values of RAM-EFR metric showed that setting cochlear filter pole functions on the basis of measured DPTHs outperforms the AudTH method for all experimental groups (Figure 7, Tables 2 and 3). This outcome is consistent with literature studies showing that OAEs are a more sensitive measure of noise-induced cochlear dysfunction in humans (Engdahl et al., 1996; Konopka et al., 2005; Marshall et al., 2009; Seixas et al., 2005). Moreover, OAEs are not influenced by inner-hair-cell/AN damage (Trautwein, 2002), whereas behaviorally measured audiometric thresholds, particularly extended highfrequency thresholds, could be affected by extreme neural degeneration (Bramhall et al., 2019; Liberman et al., 2016; Lobarinas et al., 2013). Consequently, given the varied susceptibility of AudTHs and DPOAEs to different aspects of SNHL, it was expected that we would obtain nonidentical predictions of CS profiles for a nominal subject (Table 4). Comparing the AudTH and DPTH columns in Table 4, we found a mismatch between individually predicted CS profiles for $14.28 \%$ of subjects (yNH: two, oNH: three). The mismatch degree increased to $20 \%$ (yNH: three, oNH: two and oHI: two) when the individual CS profiles were predicted using personalized classifiers $\left(\right.$ AudTH $_{\text {ind }}$ and $\mathrm{DPTH}_{\text {ind }}$ columns).

It is noteworthy that the DPTH-based cochlear individualization was implemented using DPTHs from only four frequencies $(0.8-4 \mathrm{kHz})$, whereas the AudTH-based method considered audiometric thresholds measured at 12 frequencies $(0.125-10 \mathrm{kHz})$. This difference may have resulted in less accurate CGL model parameters for the DPTH-based method, despite a better performance of forward-backward classification. In future implementations of this method, we intend to incorporate more frequencies in the DPTH measurements, especially at higher frequency regions. Employing DP-grams instead of DPTHs is another option, as these require a shorter measurement time. In both cases, we suggest to include lower stimulus levels as well, given that noise-induced OHC deficits can be identified earlier at lower stimulus levels (Bramhall et al., 2019).

\section{Method Limitations}

The proposed method for AEP-based CS profiling relies on the interactive use of recordings and model simulations. Hence, shortcomings in either aspect could have caused performance limitations of the method. The following sub-sections summarize a number of these limitations:

\section{Experimental Limitations}

(a) ABRs in humans are recorded using vertex electrodes placed on the scalp, which yields smaller and more variable wave-I amplitudes than when they are recorded in animals using subdermal electrodes. The measured ABR $\mathrm{w}-\mathrm{I}_{70}$ amplitude in our measurement produced a mean standard deviation of $0.198 \mu \mathrm{V}$ across the cohort, which is fairly large with respect to the mean amplitude of $0.146 \mu \mathrm{V}$ (yNH: $0.1964 \pm 0.1436 \mu \mathrm{V}, \quad$ oNH: $0.1304 \pm$ $0.203 \mu \mathrm{V}$, oHI: $0.1071 \pm 0.243 \mu \mathrm{V})$. Compared to $\mathrm{w}-\mathrm{I}_{70}, \mathrm{w}-\mathrm{I}_{100}$ amplitudes showed less variability, that is, $0.2503 \pm 0.2056 \mu \mathrm{V}$. Variability of the $\mathrm{w}-\mathrm{I}_{100}$ was considerably lower only for yNH group $(0.350 \pm$ $0.143 \mu \mathrm{V})$. Per subgroup, variability increased for older groups (oNH: $0.205 \pm 0.247 \mu \mathrm{V}$, oHI: $0.180 \pm 0.235 \mu \mathrm{V}$ ). Given these variabilities, adding the $\mathrm{w}-\mathrm{I}_{100}$ metric to the second feature set (RAM-EFR, $\mathrm{w}-\mathrm{V}_{\text {lat100 }}$ ), suddenly increased the $\operatorname{acc}_{\mathrm{SD}}$ (Tables 2 and 3). (b) Although adopting relative ABR metrics, such as growth functions might factor out individual differences, the standard deviation of the derived relative metric is influenced by 
the propagated error of the absolute metric. (c) ABRs to clicks presented at $100 \mathrm{~dB}$-peSPL should yield higher wave-I and V amplitudes, than when the stimulus was presented at $70 \mathrm{~dB}$-peSPL. Nevertheless, the opposite was observed in a few subjects.

\section{Model Limitations}

(a) The adopted computational model of the auditory periphery allows for $\mathrm{OHC}$ deficit simulation on a CFdependent basis, but not for CGLs above $35 \mathrm{~dB}$, since the maximum possible $\mathrm{BM}$ filter gain is $35 \mathrm{~dB}$ in the model (Verhulst et al., 2018). This constraint led to elevated absolute prediction errors for high-frequency audiometric thresholds in the oHI (above $4 \mathrm{kHz}$ ) and oNH (above $8 \mathrm{kHz}$ ) groups (Figure 4E). The increased absolute errors were mainly observed for the audiometric threshold predictions, since DPTHs were only measured for frequencies up to $4 \mathrm{kHz}$. Thus, the individualized hard-coded OHC-loss component for the oHI group might lead to similar and less accurate CS profile prediction for oHI participants with audiometric losses greater than $35 \mathrm{~dB}-\mathrm{HL}$. (b) In the adopted method, we hard-coded the CGL using the individual hearing thresholds and related the remaining AEP alterations to CS. An alternative way would be to run the model iteratively and simultaneously optimize both CGL and CS profile parameters on the basis of the experimental data to obtain the best OHC-loss and CS profiles. However, we did not further explore this route due to the high computational cost of running the adopted TL cochlear model in an iterative optimization procedure.

\section{Conclusion}

In this study, we proposed an integrated modeling and experimental approach to build personalized auditory models and predict the AN-damage profile of listeners with mixed SNHL profiles. To develop individualized cochlear models, we implemented two different methods on the basis of measured AudTHs and DPTHs. Next, we developed a classification-based approach to predict individual CS profiles and determined which AEP metric (or combinations thereof) yielded the highest prediction accuracy. Afterwards, we evaluated the implemented CGL and CS-profile individualization methods on the development data set as well as on a new cohort. Our study suggests that a DPTH-based cochlear model individualization approach combined with a RAM-EFR recording predicts individual CS profiles most accurately among the 8,191 possible combinations of 13 AEP markers. In addition, we tested the applicability of the proposed method by applying the trained classifier to the recorded RAM-EFRs of a new cohort of yNH listeners.
The classifier predicted that these listeners mostly had mild forms of CS, which supports that our method is generalizable to other recording setups and cohorts. Training the classifier again on larger cohorts may further increase the generalizability of the method. We hope that this method, or variations thereof, can be used in a clinical diagnostic context, as the number of needed AEP recordings to yield an individual CS-profile is small (i.e., $10-15 \mathrm{~min})$. Individualized models of SNHL are an important step for the development of hearing aid algorithms that compensate for both the OHC- and ANdamage aspects of SNHL.

\section{Declaration of Conflicting Interests}

The authors declared no potential conflicts of interest with respect to the research, authorship, and/or publication of this article.

\section{Funding}

The authors disclosed receipt of the following financial support for the research, authorship, and/or publication of this article: This work was supported by European Research Council under the Horizon 2020 Research and Innovation Programme, grant agreement no. 678120 RobSpear (S. K. and S. V.) and DFG Cluster of Excellence EXC 1077 1 Hearing4all (M. G. and S. V.).

\section{ORCID iDs}

Sarineh Keshishzadeh (D) https://orcid.org/0000-0003-00818243

Markus Garrett (D) https://orcid.org/0000-0002-0115-5288

\section{References}

Bharadwaj, H. M., \& Shinn-Cunningham, B. G. (2014). Rapid acquisition of auditory subcortical steady state responses using multichannel recordings. Clinical Neurophysiology, 125(9), 1878-1888. https://doi.org/10.1016/j.clinph.2014.01. 011

Bharadwaj, H. M., Verhulst, S., Shaheen, L., Liberman, M. C., \& Shinn-Cunningham, B. G. (2014). Cochlear neuropathy and the coding of supra-threshold sound. Frontiers in Systems Neuroscience, 8, 26. https://doi.org/10.3389/fnsys. 2014.00026

Boege, P., \& Janssen, T. (2002). Pure-tone threshold estimation from extrapolated distortion product otoacoustic emission i/o-functions in normal and cochlear hearing loss ears. The Journal of the Acoustical Society of America, 111(4), 1810-1818. https://doi.org/10.1121/1.1460923

Bramhall, N., Beach, E. F., Epp, B., Le Prell, C. G., LopezPoveda, E. A., Plack, C. J., Schaette, R., Verhulst, S., \& Canlon, B. (2019). The search for noise-induced cochlear synaptopathy in humans: Mission impossible? Hearing Research, 377, 88-103. https://doi.org/10.1016/j.heares. 2019.02.016

Chambers, A. R., Resnik, J., Yuan, Y., Whitton, J. P., Edge, A. S., Liberman, M. C., \& Polley, D. B. (2016). Central gain 
restores auditory processing following near-complete cochlear denervation. Neuron, 89(4), 867-879. https://doi. org/10.1016/j.neuron.2015.12.041

Chen, G.-D., Tanaka, C., \& Henderson, D. (2008). Relation between outer hair cell loss and hearing loss in rats exposed to styrene. Hearing Research, 243(1-2), 28-34. https://doi. org $/ 10.1016 / \mathrm{j}$.heares.2008.05.008

Coats, A. C., \& Martin, J. L. (1977). Human auditory nerve action potentials and brain stem evoked responses: Effects of audiogram shape and lesion location. Archives of Otolaryngology, 103(10), 605-622. https://doi.org/10.1001/ archotol.1977.00780270073012

Don, M., \& Eggermont, J. (1978). Analysis of the click-evoked brainstem potentials in man using high-pass noise masking. The Journal of the Statistical Society of America, 63(4), 1084-1092. https://doi.org/10.1121/1.381816

Elberling, C., \& Parbo, J. (1987). Reference data for ABRs in retrocochlear diagnosis. Scandinavian Audiology, 16(1), 49-55. https://doi.org/10.3109/01050398709042155

Engdahl, B., Woxen, O., Arnesen, A. R., \& Mair, I. W. (1996). Transient evoked otoacoustic emissions as screening for hearing losses at the school for military training. Scandinavian Audiology, 25(1), 71-78. https://doi.org/10. 3109/01050399609047559

Ewert, S. D., \& Dau, T. (2000). Characterizing frequency selectivity for envelope fluctuations. The Journal of the Acoustical Society of America, 108(3), 1181-1196. https:// doi.org/10.1121/1.1288665

Ewert, S. D., Kortlang, S., \& Hohmann, V. (2013). A modelbased hearing aid: Psychoacoustics, models and algorithms [Paper presentation]. Meetings on Acoustics ICA2013 (vol. 19, p. 050187). Acoustical Society of America. https://doi.org/10.1121/1.4798816

Furman, A. C., Kujawa, S. G., \& Liberman, M. C. (2013). Noise-induced cochlear neuropathy is selective for fibers with low spontaneous rates. Journal of Neurophysiology, 110(3), 577-586. https://doi.org/10.1152/jn.00164.2013

Garrett, M., \& Verhulst, S. (2019). Applicability of subcortical EEG metrics of synaptopathy to older listeners with impaired audiograms. Hearing Research, 380, 150-165. https://doi.org/10.1016/j.heares.2019.07.001

Garrett, M., Debener, S., \& Verhulst, S. (2019). Acquisition of subcortical auditory potentials with around-the-ear cEEGrid technology in normal and hearing impaired listeners. Frontiers in Neuroscience, 13, 730. https://doi.org/ $10.3389 /$ fnins.2019.00730

Garrett, M., Vasilkov, V., Mauermann, M., Wilson, J. L., Henry, K. S., \& Verhulst, S. (2020). Speech-in-noise intelligibility difficulties with age: The role of cochlear synaptopathy. bioRxiv. https://doi.org/10.1101/2020.06.09.142950

Goossens, T., Vercammen, C., Wouters, J., \& Wieringen, A. v. (2016). Aging affects neural synchronization to speechrelated acoustic modulations. Frontiers in Aging Neuroscience, 8, 133. https://doi.org/10.3389/fnagi.2016. 00133

Gorga, M. P., Worthington, D. W., Reiland, J. K., Beauchaine, K. A., \& Goldgar, D. E. (1985). Some comparisons between auditory brain stem response thresholds, latencies, and the pure-tone audiogram. Ear and Hearing,
6(2), 105-112. https://doi.org/10.1097/00003446-19850300000008

Gu, J. W., Herrmann, B. S., Levine, R. A., \& Melcher, J. R. (2012). Brainstem auditory evoked potentials suggest a role for the ventral cochlear nucleus in tinnitus. Journal of the Association for Research in Otolaryngology, 13(6), 819-833. https://doi.org/10.1007/s10162-012-0344-1

Guest, H., Munro, K. J., Prendergast, G., Millman, R. E., \& Plack, C. J. (2018). Impaired speech perception in noise with a normal audiogram: No evidence for cochlear synaptopathy and no relation to lifetime noise exposure. Hearing Research, 364, 142-151. https://doi.org/10.1016/j.heares. 2018.03.008

Heinz, M. G., Zhang, X., Bruce, I. C., \& Carney, L. H. (2001). Auditory nerve model for predicting performance limits of normal and impaired listeners. Acoustics Research Letters Online, 2(3), 91-96. https://doi.org/10.1121/1.1387155

Henry, K. S., \& Abrams, K. S. (2018). Persistent auditory nerve damage following kainic acid excitotoxicity in the budgerigar (melopsittacus undulatus). Journal of the Association for Research in Otolaryngology, 19(4), 435-449. https://doi.org/10.1007/s10162-018-0671-y

Herdman, A. T., \& Stapells, D. R. (2003). Umbrales de las respuestas auditivas de estado estable en adultos con hipoacusia sensorineural [Auditory steady-state response thresholds of adults with sensorineural hearing impairments]. International Journal of Audiology, 42(5), 237-248. https:// doi.org/10.3109/14992020309078343

Hickox, A. E., \& Liberman, M. C. (2014). Is noise-induced cochlear neuropathy key to the generation of hyperacusis or tinnitus? Journal of Neurophysiology, 111(3), 552-564. https://doi.org/10.1152/jn.00184.2013

Hickox, A. E., Larsen, E., Heinz, M. G., Shinobu, L., \& Whitton, J. P. (2017). Translational issues in cochlear synaptopathy. Hearing Research, 349, 164-171. https://doi.org/ 10.1016/j.heares.2016.12.010

Jepsen, M. L., \& Dau, T. (2011). Characterizing auditory processing and perception in individual listeners with sensorineural hearing loss. The Journal of the Acoustical Society of America, 129(1), 262-281. https://doi.org/10.1121/1. 3518768

Jepsen, M. L., Ewert, S. D., \& Dau, T. (2008). A computational model of human auditory signal processing and perception. The Journal of the Acoustical Society of America, 124(1), 422-438. https://doi.org/10.1121/1.2924135

Keshishzadeh, S., \& Verhulst, S. (2019). From derived-band envelope-following responses to individualized models of near-and supra-threshold hearing deficits [Paper presentation]. Proceedings of the International Symposium on Auditory and Audiological Research (vol. 7, pP. 13-20). https://proceedings.isaar.eu/index.php/isaarproc/article/ view/2019-02

Keshishzadeh, S., Garrett, M., Vasilkov, V., \& Verhulst, S. (2020). The derived-band envelope following response and its sensitivity to sensorineural hearing deficits. Hearing Research, 107979. https://doi.org/10.1016/j.heares.2020. 107979

Konopka, W., Pawlaczyk-Luszczynska, M., SliwinskaKowalska, M., Grzanka, A., \& Zalewski, P. (2005). 
Efectos del ruido impulsivo sobre las emisiones otoacústicas evocadas por transitorios en soldados [Effects of impulse noise on transiently evoked otoacoustic emission in soldiers]. International Journal of Audiology, 44(1), 3-7. https://doi.org/10.1080/14992020400022561

Kujawa, S. G., \& Liberman, M. C. (2009). Adding insult to injury: Cochlear nerve degeneration after "temporary" noise-induced hearing loss. Journal of Neuroscience, 29(45), 14077-14085. https://doi.org/10.1523/JNEUROSCI.2845-09. 2009

Kummer, P., Janssen, T., \& Arnold, W. (1998). The level and growth behavior of the $2 \mathrm{f} 1-\mathrm{f} 2$ distortion product otoacoustic emission and its relationship to auditory sensitivity in normal hearing and cochlear hearing loss. The Journal of the Acoustical Society of America, 103(6), 3431-3444. https://doi.org/10.1121/1.423054

Liberman, M. C. (1978). Auditory-nerve response from cats raised in a low-noise chamber. The Journal of the Acoustical Society of America, 63(2), 442-455. https://doi. org/10.1121/1.381736

Liberman, M. C., Epstein, M. J., Cleveland, S. S., Wang, H., \& Maison, S. F. (2016). Toward a differential diagnosis of hidden hearing loss in humans. PLoS One, 11(9): e0162726. https://doi.org/10.1371/journal.pone.0162726

Lobarinas, E., Salvi, R., \& Ding, D. (2013). Insensitivity of the audiogram to carboplatin induced inner hair cell loss in chinchillas. Hearing Research, 302, 113-120. https://doi. org/10.1016/j.heares.2013.03.012

Long, G. R., Talmadge, C. L., \& Lee, J. (2008). Measuring distortion product otoacoustic emissions using continuously sweeping primaries. The Journal of the Acoustical Society of America, 124(3), 1613-1626. https://doi.org/10.1121/1. 2949505

Marshall, L., Lapsley Miller, J. A., Heller, L. M., Wolgemuth, K. S., Hughes, L. M., Smith, S. D., \& Kopke, R. D. (2009). Detecting incipient inner-ear damage from impulse noise with otoacoustic emissions. The Journal of the Acoustical Society of America, 125(2), 995-1013. https://doi.org/10. $1121 / 1.3050304$

Mauermann, M. (2013). Improving the usability of the distortion product otoacoustic emissions (DPOAE)-sweep method: An alternative artifact rejection and noise-floor estimation [Paper presentation]. Meetings on Acoustics ICA2013 (vol. 19, p. 050054). Acoustical Society of America. https://doi.org/10. $1121 / 1.4800902$

Mitchell, C., Phillips, D. S., \& Trune, D. R. (1989). Variables affecting the auditory brainstem response: Audiogram, age, gender and head size. Hearing Research, 40(1-2), 75-85. https://doi.org/10.1016/0378-5955(89)90101-9

Möhrle, D., Ni, K., Varakina, K., Bing, D., Lee, S. C., Zimmermann, U., Knipper, M., \& Rüttiger, L. (2016). Loss of auditory sensitivity from inner hair cell synaptopathy can be centrally compensated in the young but not old brain. Neurobiology of Aging, 44, 173-184. https://doi.org/ 10.1016/j.neurobiolaging.2016.05.001
Osses, A., \& Verhulst, S. (2019). Calibration and reference simulations for the auditory periphery model of Verhulst et al. 2018 version 1.2. arXiv preprint arXiv, 1912.10026.

Parthasarathy, A., \& Kujawa, S. G. (2018). Synaptopathy in the aging cochlea: Characterizing early-neural deficits in auditory temporal envelope processing. Journal of Neuroscience, 38(32), 7108-7119. https://doi.org/10.1523/ JNEUROSCI.3240-17.2018

Parthasarathy, A., Bartlett, E. L., \& Kujawa, S. G. (2019a). Age-related changes in neural coding of envelope cues: Peripheral declines and central compensation. Neuroscience, 407, 21-31. https://doi.org/10.1016/j.neurosci ence.2018.12.007

Parthasarathy, A., Herrmann, B., \& Bartlett, E. L. (2019b). Aging alters envelope representations of speech-like sounds in the inferior colliculus. Neurobiology of aging, 73, 30-40. https://doi.org/10.1016/j.neurobiolaging.2018. 08.023

Plack, C. J., Léger, A., Prendergast, G., Kluk, K., Guest, H., \& Munro, K. J. (2016). Toward a diagnostic test for hidden hearing loss. Trends in Hearing, 20, 2331216516657466. https://doi.org/10.1177/2331216516657466

Prendergast, G., Tu, W., Guest, H., Millman, R. E., Kluk, K., Couth, S., Munro, K. J., \& Plack, C. J. (2018). Suprathreshold auditory brainstem response amplitudes in humans: Test-retest reliability, electrode montage and noise exposure. Hearing Research, 364, 38-47. https://doi. org/10.1016/j.heares.2018.04.002

Purcell, D. W., John, S. M., Schneider, B. A., \& Picton, T. W. (2004). Human temporal auditory acuity as assessed by envelope following responses. The Journal of the Acoustical Society of America, 116(6), 3581-3593. https:// doi.org/10.1121/1.1798354

Rohdenburg, T., Hohmann, V., \& Kollmeier, B. (2005). Objective perceptual quality measures for the evaluation of noise reduction schemes [Paper presentation]. 9th International Workshop on Acoustic Echo and Noise Control (pp. 169-172).

Schaette, R., \& McAlpine, D. (2011). Tinnitus with a normal audiogram: Physiological evidence for hidden hearing loss and computational model. Journal of Neuroscience, 31(38), 13452-13457. https://doi.org/10.1523/JNEUROSCI.215611.2011

Seixas, N., Goldman, B., Sheppard, L., Neitzel, R., Norton, S., \& Kujawa, S. (2005). Prospective noise induced changes to hearing among construction industry apprentices. Occupational and Environmental Medicine, 62(5), 309-317. http://dx.doi.org/10.1136/oem.2004.018143

Sergeyenko, Y., Lall, K., Liberman, M. C., \& Kujawa, S. G. (2013). Age-related cochlear synaptopathy: An early-onset contributor to auditory functional decline. Journal of Neuroscience, 33(34), 13686-13694. https://doi.org/10. 1523/JNEUROSCI.1783-13.2013

Shaheen, L. A., Valero, M. D., \& Liberman, M. C. (2015). Towards a diagnosis of cochlear neuropathy with envelope following responses. Journal of the Association for Research 
in Otolaryngology, 16(6), 727-745. https://doi.org/10.1007/ s10162-015-0539-3

Stamper, G. C., \& Johnson, T. A. (2015). Auditory function in normal-hearing, noise-exposed human ears. Ear and Hearing, 36(2), 172. https://doi.org/10.1097/AUD.0000000000000107

Trautwein, P. (2002, November 8-10). Auditory neuropathy: Diagnosis and case management [Paper presentation]. 4th ACFOS International Conference (pp. 8-10, Paris, France).

Trune, D. R., Mitchell, C., \& Phillips, D. S. (1988). The relative importance of head size, gender and age on the auditory brainstem response. Hearing Research, 32(2-3), 165-174. https://doi.org/10.1016/0378-5955(88)90088-3

Valero, M., Burton, J., Hauser, S., Hackett, T., Ramachandran, R., \& Liberman, M. (2017). Noise-induced cochlear synaptopathy in rhesus monkeys (Macaca mulatta). Hearing Research, 353, 213-223. https://doi.org/ 10.1016/j.heares.2017.07.003

Vasilkov, V., \& Verhulst, S. (2019). Towards a differential diagnosis of cochlear synaptopathy and outer-hair-cell deficits in mixed sensorineural hearing loss pathologies. medRxiv, 19008680. https://doi.org/10.1101/19008680

Vasilkov, V., Garrett, M., Mauermann, M., \& Verhulst, S. (2021). Enhancing the sensitivity of the envelope-following response for cochlear synaptopathy screening in humans: The role of stimulus envelope. Hearing Research, 400, 108132. https://doi.org/10.1016/j.heares.2020.108132

Verhulst, S., Altoe, A., \& Vasilkov, V. (2018). Computational modeling of the human auditory periphery: Auditory-nerve responses, evoked potentials and hearing loss. Hearing
Research, 360, 55-75. https://doi.org/10.1016/j.heares. 2017.12.018

Verhulst, S., Dau, T., \& Shera, C. A. (2012). Nonlinear timedomain cochlear model for transient stimulation and human otoacoustic emission. The Journal of the Acoustical Society of America, 132(6), 3842-3848. https://doi.org/10. $1121 / 1.4763989$

Verhulst, S., Jagadeesh, A., Mauermann, M., \& Ernst, F. (2016). Individual differences in auditory brainstem response wave characteristics: Relations to different aspects of peripheral hearing loss. Trends in Hearing, 20, 2331216516672186. https://doi.org/10.1177/2331216516672186

Watson, D. R. (1996). The effects of cochlear hearing loss, age and sex on the auditory brainstem response. Audiology, 35(5), 246-258. https://doi.org/10.3109/00206099609071945

Wu, P.-Z., O’Malley, J. T., de Gruttola, V., \& Liberman, M. C. (2020). Age-related hearing loss is dominated by damage to inner ear sensory cells, not the cellular battery that powers them. Journal of Neuroscience, 40(33), 6357-6366. https:// doi.org/10.1523/JNEUROSCI.0937-20.2020

Zhu, L., Bharadwaj, H., Xia, J., \& Shinn-Cunningham, B. (2013). A comparison of spectral magnitude and phaselocking value analyses of the frequency-following response to complex tones. The Journal of the Acoustical Society of America, 134(1), 384-395. https://doi.org/10.1121/1.4807498

Zilany, M. S., \& Bruce, I. C. (2006). Modeling auditory-nerve responses for high sound pressure levels in the normal and impaired auditory periphery. The Journal of the Acoustical Society of America, 120(3), 1446-1466. https://doi.org/10. $1121 / 1.2225512$ 